
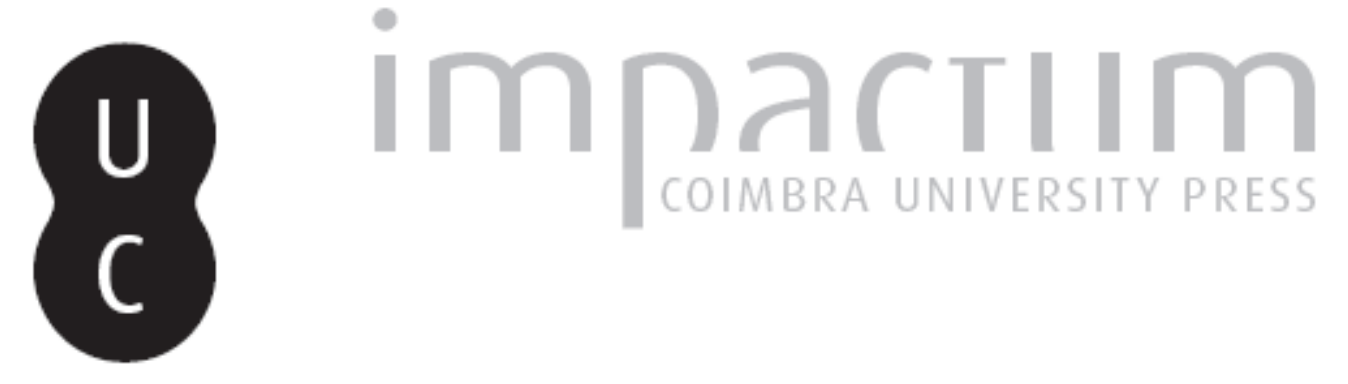

\title{
Les vettons et la mort: aux trois premiers siècles de notre ère
}

Autor(es): $\quad$ Bonnaud, Christophe

Publicado por: Faculdade de Letras da Universidade de Coimbra

URL persistente:

URI:http://hdl.handle.net/10316.2/37808

DOI:

DOI:http://dx.doi.org/10.14195/1647-8657_44_2

Accessed : $\quad$ 26-Apr-2023 13:34:28

A navegação consulta e descarregamento dos títulos inseridos nas Bibliotecas Digitais UC Digitalis, UC Pombalina e UC Impactum, pressupõem a aceitação plena e sem reservas dos Termos e Condições de Uso destas Bibliotecas Digitais, disponíveis em https://digitalis.uc.pt/pt-pt/termos.

Conforme exposto nos referidos Termos e Condições de Uso, o descarregamento de títulos de acesso restrito requer uma licença válida de autorização devendo o utilizador aceder ao(s) documento(s) a partir de um endereço de IP da instituição detentora da supramencionada licença.

Ao utilizador é apenas permitido o descarregamento para uso pessoal, pelo que o emprego do(s) título(s) descarregado(s) para outro fim, designadamente comercial, carece de autorização do respetivo autor ou editor da obra.

Na medida em que todas as obras da UC Digitalis se encontram protegidas pelo Código do Direito de Autor e Direitos Conexos e demais legislação aplicável, toda a cópia, parcial ou total, deste documento, nos casos em que é legalmente admitida, deverá conter ou fazer-se acompanhar por este aviso.

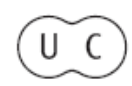




\section{CONIMBRIGA}

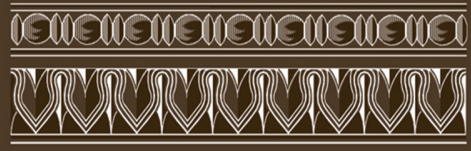

INSTITUTO DE ARQUEOLOGIA

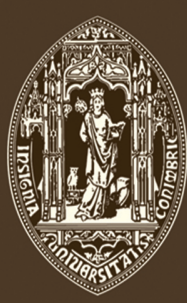

VOLUME XLIV • 2005

FACULDADE DE LETRAS UNIVERSIDADE DE COIMBRA 
Christophe Bonnaud

Professeur d'Histoire au Lycée Français du Caire. Docteur en Histoire ancienne.

LES VETTONS ET LA MORT

AUX TROIS PREMIERS SIÈCLES DE NOTRE ÈRE

"Conimbriga" XLIV (2005) p. 25-68

RÉsumÉ: L'ouest de la Meseta espagnole (principalement les provinces de Salamanque, Cáceres, Ávila) ainsi qu'une mince bande de terre portugaise étaient occupés dans l'Antiquité par le peuple des Vettons. Ceux-ci nous ont laissé une abondante documentation épigraphique, en grande partie composée de stèles funéraires datant presque toutes du Haut-Empire romain (Ier - IIIè siècles). En dépit des problèmes de datation, d'interprétation des motifs et de transcription des épitaphes, ces stèles se révèlent être de précieux outils pour étudier les mentalités et les pratiques funéraires des Vettons, sur lesquelles les textes littéraires sont pratiquement muets. Mais ces stèles proviennent de nécropoles très mal localisées et dont le contexte archéologique demeure problématique. Il est également nécessaire de s'attarder sur la forme des stèles, des motifs symboliques et/ou décoratifs mais aussi sur les épitaphes qui constituent la partie essentielle des stèles funéraires. Ces épitaphes comprennent différents éléments qui nous permettent de mieux saisir la mentalité funéraire des Vettons et qui nous renseignent aussi sur le milieu social des défunts. Enfin, une particularité de la culture des Vettons réside dans l'existence de verracos, ces massives sculptures zoomorphes, dont certaines semblent avoir été utilisées à des fins funéraires. Comment l'archéologie et l'épigraphie témoignent-elles de cette pratique peu commune? 
(Página deixada propositadamente em branco) 


\section{LES VETTONS ET LA MORT AUX TROIS PREMIERS SIÈCLES DE NOTRE ÈRE}

Les inscriptions funéraires du Haut-Empire représentent plus des trois quarts des documents épigraphiques vettons. Un décompte précis n'a cependant pas encore vu le jour, malgré les progrès importants réalisés ces dernières années dans le domaine de l'épigraphie retrouvée sur le territoire de cette population. En effet, nombre de recueils et corpus ont récemment paru mais aucun ne s'attache à un recensement épigraphique ayant pour cadre la Vettonnie antique (CRESPO et AlONSO 1999 pour le territoire de Castilla y León; ALONSO et CRESPO 1999 pour la province de Salamanque; SÁNCHEZ et VINAGRE 1998, pour le territoire de Coria; Solana et Hernández 2000, pour la Meseta septentrionale). Il est certain qu'à l'intérieur du conventus Emeritensis, les limites de la Vettonia du Haut-Empire se caractérisent par une réelle imprécision que nous avions essayé de réduire dans un travail précédent (BONNAUD 2002 171-199). Rappelons brièvement ici que ce territoire s'étendait sur la plus grande partie des provinces espagnoles de Salamanque et d'Ávila, la moitié orientale de celle de Cáceres, avec des appendices sur les provinces de Tolède et Badajoz, peut-être Zamora, ainsi que sur une frange orientale du Portugal.

A partir de cette constatation, toute tentative de décompte de la documentation épigraphique des Vettons ne peut que se heurter à ce problème de délimitation précise mais il ne fait aucun doute que les documents en question dépassent le millier, l'essentiel provenant de la province de Salamanque (Alonso et CRESPO 1999, en ont recensé 340, sans tenir compte de la zone de Hinojosa de Duero) et de la partie orientale de la province de Cáceres. Nous avons donc à notre disposition un matériel considérable, ayant pour cadre chronologique, à quelques exceptions près, les trois premiers siècles de notre ère, cette période correspondant dans les provinces de l'empire romain, à l'éclosion d'un "mouvement épigraphique" en relation avec la pax romana instaurée 
par Auguste et l'intégration des mondes indigènes aux modes d'expression de la romanité (pour une tentative de datation plus précise des stèles avilaises, à partir de différents critères, $c f$. KNAPP 1992 339-383; sur les origines du phénomène épigraphique dans l'ouest de la Meseta, $c f$. SALINAS 1995 281-282). Tout ce matériel est à même de nous fournir des informations sur les pratiques et le symbolisme funéraire des Vettons du Haut-Empire. Reste à savoir si ces inscriptions nous permettent d'avoir une connaissance détaillée des liens qui pouvaient exister entre les Vettons et le monde des morts. En outre, en l'absence de sources littéraires évoquant, même de façon très indirecte, les pratiques funéraires des Vettons, il semble indispensable d'avoir une vision plus élargie et de ne jamais perdre de vue les coutumes funéraires des peuples voisins des Vettons, ces derniers n'ayant par ailleurs jamais constitué une entité ethnique culturellement repliée sur elle-même. Ainsi, nous avons constaté que le panthéon lusitanien a eu une influence considérable en Vettonnie (jusqu'à Postoloboso, province d'Ávila, avec le culte de Vaelicus par exemple). En ce sens, il existait une rélle "communauté de culte" entre Lusitaniens et Vettons (BONNAUD 2002a). Il est certain que cette "communauté" devait se retrouver également dans le domaine funéraire.

\section{Les nécropoles}

\section{Une grave lacune dans la recherche: leur localisation}

Il peut sembler paradoxal de constater qu'en Vettonnie, les nécropoles des castros du $2 \mathrm{~d}$ Âge du Fer sont mieux connues que celles de l'époque romaine (pour une synthèse de l'archéologie des nécropoles du 2d Âge du Fer avec la bibliographie, $c f$. ÁlvarEZ-SANCHÍs 1999 169-213). En fait, le paradoxe n'est qu'apparent car nous savons où se trouvent nombre de nécropoles protohistoriques en raison de l'abandon plus ou moins immédiat à la conquête romaine de quelques castros. Dans ce cas, il est possible par voie de sondage de localiser la ou les nécropoles (extérieures à l'enceinte) de ces castros quand la tradition orale ne permet aucune localisation. La situation est déjà différente lorsque le castro a perduré sous la domination romaine car les nécropoles protohistoriques ont pu continuer d'être utilisées par la population locale, ou bien être délaissées au profit d'une ou plusieurs autres nécropoles pas tou- 
jours aisément localisables du fait de témoignages funéraires plus discrets (modification des pratiques funéraires), peut-être plus aisément destructibles, et aussi en raison de la dispersion des stèles funéraires au point de ne plus savoir d'où elles proviennent précisément. Lorsque le castro a perduré, souvent jusqu'à nos jours comme c'est le cas de plusieurs villes vettonnes (Salamanque, Trujillo, Talavera de la Reina...), la localisation des nécropoles est encore plus problématique car leur emplacement risque d'être recouvert par le développement de la ville.

L'idée de l'inexistence de véritables nécropoles, d'une pratique totalement individuelle ou familiale de l'inhumation ou de l'incinération (consistant à garder près de soi ou dans un endroit isolé une seule ou un petit groupe de tombes) n'est guère convaincante car une telle pratique serait alors unique dans toute la péninsule ibérique, voire dans l'ensemble du monde romain (MORRIS, 1992; Incinérations et inhumations 1992; La mort des notables en Gaule romaine 2002).

Le problème de la localisation des nécropoles de l'époque romaine est tel que la recherche n'a pas permis, jusqu'à aujourd'hui, de déterminer l'emplacement précis des lieux collectifs de sépultures. Dans certains cas comme à Caesarobriga nous ignorons même la zone où devaient se situer les nécropoles; nous disposons d'indices plus nombreux, bien que problématiques, en ce qui concerne des noyaux de peuplement tels que Salamanque, Ciudad Rodrigo, Las Merchanas, Yecla de Yeltes, Hinojosa de Duero, Trujillo, Capera, Lacimurga.

A Talavera de la Reina, les quelque 80 documents épigraphiques étaient intégrés aux murs de vieux couvents ou palais aristocratiques, comme par exemple l'ancien palais des Pères Jésuites ou l'ermitage de Santa Maria del Prado, à des églises, à des fontaines, à des maisons particulières ou encore à la muraille de la ville (CIL II 893-947 5315-5342; Cedillo 1959 287-345; CoRTES HernándeZ et alii 1989-1990 67-77). Il serait fort imprudent de déduire leur provenance d'origine d'après une localisation postérieure répondant à un souci d'esthétique et de prestige. Toutes ces stèles ont été ramenées d'un ou plusieurs endroits sans doute à proximité immédiate de la ville (MANGAS et CARROBLES 1992 109-110). Des sondages archéologiques seraient les bienvenus, en particulier en bordure du Tage qui a pu recouvrir tout ou partie de la zone funéraire, comme ce fut le cas dans la ville voisine d'Augustobriga.

Nous sommes à peine plus renseignés sur la nécropole de Turgalium, dont même l'urbanisme antique nous est inconnu. Le fait que 
nombre de stèles proviennent du château n'est pas, là non plus, l'indice de la proximité d'une nécropole. L'hypothèse la plus probable est de localiser une zone funéraire à Aldehuela de Mordazo car un autre groupe de stèles funéraires provient de ce hameau proche de la ville, au-delà de la rivière Tamuja, sur la route de Badajoz. De plus, on y a retrouvé de grandes tuiles (probablement à usage funéraire) et les restes d'une sépulture en granite, de forme rectangulaire, mais qui ne sont peut-être pas contemporains des stèles (BELTRÁN 1975-1976 43). Nous sommes incontestablement en présence d'un lieu funéraire mais il est situé à environ deux kilomètres de la ville. S'agit-il d'une des nécropoles de Turgalium ou celle d'un hameau proche de la ville? Tout noyau de peuplement, hameau ou villa, comportait en effet une nécropole et on peut être tenté de rattacher aux villes vettonnes, dont l'étendue demeure mal connue, des nécropoles rurales.

Un problème semblable se pose dans la cité de Lacimurga où ont été mises au jour, au lieu-dit Setecientas, les restes d'une nécropole d'époque romaine. Mais nous nous situons sur les rives du lac de barrage d'Orellana et une partie de la nécropole a été inondée, tandis que l'autre a été victime de pillage. Décrite par A. Aguilar et P. Guichard, cette importante nécropole a livré des tombes délimitées par des tuiles verticales, des fragments de tuiles et de céramique commune et des morceaux de verre provenant du mobilier des sépultures (AGUILAR et GUICHARD 1993 60). S'agit-il de la nécropole de Lacimurga ou de celle d'un des innombrables sites ruraux environnants? A en juger par l'importance des vestiges, on serait tenté de privilégier la première hypothèse bien qu'une nécropole rurale ait pu être utilisée par les habitants de plusieurs fermes et villas (sur la richesse de l'occupation du sol dans cette cité $c f$. Aguilar et Guichard 1995; Aguilar Guichard et LeFEBVRE 1994 109-130). On peut s'interroger sur la localisation du noyau de Lacimurga par rapport à cette nécropole. Si on admet qu'elle se situait bien à Cogolludo, la nécropole se trouve à environ deux kilomètres de la "ville", de l'autre côté du fleuve (Guadiana) mais sur la commune d'Esparragosa; on constate donc la même distance du noyau urbain, le même type de localisation qu'à Trujillo.

A Talavera de la Vieja (l'Augustobriga romaine), un auteur du XVIIIè siècle évoque des traces de sépultures et d'ossements retrouvées par les paysans au bord du Tage (MORÁN SÁNCHEZ 18). En 1952, une vigne a livré des tombes composées de briques et de mortier de chaux. Au même endroit des bustes romains ont été découverts. Beaucoup plus 
récemment, en 1993, la décrue des eaux du fleuve a permis la mise au jour, dans une oliveraie, de plusieurs sépultures recouvertes de tuiles et pierres de granite. La brève description que nous en avons permet d'envisager une datation romaine, sans autres précision et sans certitude (SANTOS SÁnchez 1993).

A Capera, A. Floriano avait localisé à l'extérieur des murs de la ville trois nécropoles: deux à incinération (au sud-est, près de l'entrée de la ville, et au nord-ouest) et une à inhumation (au sud-ouest) (FLORIANO 1944 283-286). Cet auteur suspectait néanmoins, mais sans exposer ses éléments d'appréciation, l'existence d'une quatrième nécropole que ni J. M. Blázquez ni E. Cerrillo ne sont parvenus semble-t-il, à localiser (BLÁZQuEZ 1965 17-19).

A Salamanque, la situation est encore moins claire. Les fouilles les plus récentes ont rendu caduques les hypothèses formulées en son temps par J. Maluquer. Celui-ci croyait pouvoir déceler la présence d'une nécropole protohistorique au Cerro de la Peña, ensuite intégrée à la ville romaine, et la présence d'une nécropole du Bas-Empire au sud du Cerro de San Vicente (MALUQUER 1951 71). La provenance véritable des stèles funéraires du Haut-Empire mises au jour dans la ville, en particulier dans la zone orientale de la ville (50\%), est inconnue. On suppose toutefois qu'elles pourraient provenir d'une zone funéraire située à l'est de la muraille à laquelle certaines d'entre elles auraient été incorporées (MARTín VALLS et alii 1992 112). Le doute est néanmoins permis, surtout en l'absence de fouilles archéologiques dans cette zone. Quant à la présence d'un torico à proximité du pont romain, cela ne peut constituer la preuve de l'existence toute proche d'une nécropole car cette sculpture, même en admettant une fonction funéraire, a pu être déplacée depuis un lieu éloigné du pont. La sculpture était cependant déjà à cet emplacement lorsque le Lazarillo de Tormes fut rédigé.

La localisation des nécropoles de Ciudad Rodrigo est tout aussi incertaine, tout comme la délimitation même du noyau romain. Deux stèles funéraires étant intégrées à un mur du couvent San Francisco, R. Martín Valls a supposé qu'une nécropole devait se trouver dans cette partie de la ville (MARTín VALLS 1976 386-387). Mais des stèles anépigraphes gisaient près du pont et certaines ont dû servir à sa construction; une nécropole a donc pu exister à l'ouest de la ville. R. Martín Valls s'appuie d'ailleurs sur la présence d'un verraco ("à finalité funéraire?”) près du pont pour suggérer la proximité d'une nécropole 
(Martín Valls 1976 386; SÁnchez Cabañas 1967 51). Nous verrons ultérieurement s'il faut considérer tous les verracos comme des sculptures provenant de nécropoles.

Dans l'ouest de la province de Salamanque, le castro de Las Merchanas perdurant sous la domination romaine a livré une nécropole à inhumation qui a été en partie fouillée, entre la muraille nord du castro et la limite du territoire de Lumbrales (MALUQUER 1968 115-128). Il est surprenant de noter l'éloignement de la porte principale mais il faut rappeler qu'il s'agit d'une nécropole du Bas-Empire (IVè-Vè siècles), celle du Haut-Empire n'ayant pas été localisée, malgré des sondages effectués à différents endroits, et pouvant se trouver plus près de l'entrée principale (MALUQUER 196810 107). De plus, l'accès à la nécropole de Las Merchanas pouvait se faire par une entrée protégée par ce qui ressemble à une tour massive. L'étendue totale de la zone funéraire est difficile à estimer car seules 36 tombes ont été dégagées et on voit mal pourquoi la nécropole ne se serait pas étendue sur le territoire voisin de Bermellar.

Le site de Yecla de Yeltes, également dans l'ouest salmantin, est intéressant en raison de la longue durée d'occupation de ce castro qui a fait usage de plusieurs nécropoles (MARTín VALLS 1979 499-501; MARTín VALLS 1982 181-182). La première nécropole (à incinération) se trouverait à quelques mètres de la porte principale au nord du castro et devait être contemporaine de celles de Las Cogotas ou de La Osera ( 2 d Âge du Fer). La nécropole du Haut-Empire (non loin de la précédente) a dû continuer le rite de l'incinération. Les stèles funéraires correspondant à cette nécropole (du Ier au IIIè siècles) ont cependant été retrouvées assez loin de cet endroit, dispersées en trois lieux différents, tout particulièrement au lieu-dit La Verdadera, une petite hauteur située à moins d'un kilomètre au nord du castro, où pouvait se trouver la nécropole du Haut-Empire. Enfin, la troisième nécropole, plus tardive et très étendue, comportait des tombes composées de stèles faisant office de parois latérales. Sur ces trois nécropoles, seule celle du BasEmpire a été localisée avec certitude.

A Ávila, l'escarpement qui entoure les côtés nord, ouest et sud de la ville permet de restreindre le champ des recherches. C'est bien à l'est de la ville, dans une zone relativement plane, qu'il faut situer la ou les nécropoles principales de l'Avela romaine (RoDRíguez AlmEIDA 1980 46-47). Cette localisation est en outre attestée par la proximité de la basilique sépulcrale et surtout par le grand nombre de stèles et éléments 
architecturaux funéraires (cistae, cupae semi-cylindriques) contenus dans la partie orientale de la muraille, notamment dans la puerta de San Vicente. Une grande partie de la calle San Segundo, qui longe le pan oriental de la muraille, comporte des constructions qui s'appuient sur cette partie de l'enceinte et qui cachent sans le moindre doute d'autres pièces funéraires intéressantes. Comme le rappelle E. Rodríguez Almeida, d'autres lieux de sépultures, de moindre importance pour des raisons topographiques devaient exister ailleurs qu'à proximité de la puerta de San Vicente, en particulier vers la puerta de San Segundo, qui donne sur le pont et où la muraille contient une autre concentration de stèles, et peut-être aussi près des puertas de Montenegro et Gonzalo Dávila (RodríGuez AlmeIda 1980 47). Ainsi, la coutume des nécropoles traversées par les voies pénétrant en ville, serait ici respectée (LAFON 2002 113-120).

\section{Incinération et inhumation}

Il est nécessaire de revenir très brièvement sur quelques données d'archéologie funéraire pour une meilleure compréhension des rites pratiqués en Vettonnie.

Dans la péninsule ibérique, on constate dans certaines régions une vieille tradition d'inhumation, attestée notamment dans le sud péninsulaire, et peut-être également dans le pays vetton (BENDALA GALÁN 1991 80-81). Ainsi, dans la nécropole d'incinération de La Osera, les urnes funéraires se présentaient sous la forme de tumuli et étaient déposées à l'intérieur d'autres tumuli constitués de pierres circulaires (CABRÉ et alii 1950 43ss). Cela pourrait révéler la survivance d'une très ancienne pratique d'inhumation issue du fonds préceltique. L'influence des "Champs d'urnes" dans la Meseta aurait introduit le rite de l'incinération mais sans détruire totalement et aussitôt celui de l'inhumation (TARradell 1980 157; Marco Simón 1991 92-101; Fuentes DomínGUEZ 1991 96). A partir de la fin de la République, apparaît dans la péninsule la coutume de l'inhumation, bien que là encore les deux rites coexistent quelquefois longtemps (sur la "romanisation" progressive des coutumes funéraires en pays celtibère, $c f$. CuRCHIN 1997 7-34). Au IIIè siècle ap. J.C., l'incinération semble avoir presque totalement disparu de la péninsule (FuENTES DomínGueZ 1991 99). 


\section{L’incinération dans la Vettonnie du Haut-Empire}

Les deux nécropoles d'incinération de Capera sont les seules zones funéraires urbaines de Vettonnie à nous fournir des témoignages in situ. La première nécropole (sud-est de la ville) a fourni une tombe comprenant une fosse de pierres, de terre, et, dans sa partie supérieure, de briques, le tout clos par une grosse pierre. Dans cette fosse peu profonde $(0,26 \mathrm{~m})$, on avait placé une urne cinéraire, sans doute en terre, et dont il ne subsistait, au moment de la fouille, que quelques pièces de fer et de bronze qui devaient faire partie du système de fermeture de l'urne (FLORIANO 1944 284-285).

L'autre nécropole (nord-ouest) a livré une seule autre tombe d'incinération, assez différente de la précédente. En effet, l'urne a été taillée dans un bloc de pierre, tout aussi peu profondément que dans la première sépulture $(0,22 \mathrm{~m})$ et était fermée par un morceau d'ardoise. L'urne, contenant les restes calcinés, était enterrée dans une simple fosse creusée dans le sol (FLORIANO 1944 285).

La chronologie de ces deux sépultures est difficile à préciser: très certainement le Haut-Empire, du moins pour l'une d'entre elles (à en juger par le mobilier funéraire contenu dans la première tombe décrite, datant du Ier siècle ap. J.C.) (BLÁZQuez 1965 18). Mais des questions subsistent: les deux emplacements funéraires correspondent-ils à une différence sociale des défunts (mobilier funéraire plus riche dans la première tombe) ou à un agrandissement nécessaire, à une date postérieure, d'une des deux nécropoles? La deuxième sépulture décrite fait-elle partie d'une nécropole protohistorique? Comment expliquer le relatif éloignement entre les deux zones funéraires? De telles questions risquent de rester sans réponses en l'absence de fouilles qui viendraient compléter celles déjà anciennes (dans les années 1920), d'A. Floriano.

Le rituel de l'incinération nous est relativement bien connu, en particulier en Gaule romaine (AUDIN 1960 312-322 et 518-532; HATT 1986 65ss; Coulon juin 1987 26-32; Bel, Tranoy, Beraud et Gebara 1992 9-29; Bost et MAURIN 1992 41-65; TrAnOy 2002 105-107). Nous savons qu'il comportait plusieurs étapes et qu'il ne se limitait pas à brûler le défunt avec ses objets personnels, à renfermer ses restes dans une urne, et à déposer des objets personnels dans la tombe. La sépulture pouvait en outre être l'objet de libations funèbres (fusiones). Celles-ci consistaient à faire passer des liquides jusqu'aux cendres des défunts grâce à un aménagement spécial (ouverture, canalisation) de la sépul- 
ture. Ce dispositif est notamment attesté à Mérida (WoLSKI et BERCIU 1973 371-379; Molano Brías et Alvarado GonZÁlo 1991-1992 161-173).

Les deux nécropoles de Capera n'ont livré aucun élément susceptible d'être mis en rapport avec cette pratique. Mais à Ávila des blocs de pierre scellés à la muraille ont été taillés de telle manière qu'ils ont dû faire partie d'un dispositif pour libations (RoDRíGUEZ ALMEIDA 1980 87-90). Il s'agit de blocs rectangulaires (cistae) creusés dans leur partie centrale qui forme alors une fosse (5 à $10 \mathrm{~cm}$ de profondeur) également rectangulaire $(0,30 \times 0,20 \mathrm{~m})$. Cette fosse communique au moyen d'un petit canal (infundibulum) jusqu'au bord de la cista et se termine en petits puits peu profond. C'est par cette ouverture que le liquide parvenait jusqu'aux cendres des défunts. La fosse devait être recouverte d'une cupa, ou bloc de coupe semi-circulaire sur lequel était gravée l'épitaphe. De nombreuses cistae et quelques cupae sont reconnaissables dans la muraille d'Ávila. Un nombre sans doute beaucoup plus grand n'est pas identifiable en raison d'un mauvais état de conservation ou de la position des pierres (face permettant de les reconnaître vers l'intérieur de la muraille). Malheureusement, ces éléments d'architecture funéraire sont totalement en dehors de leur contexte archéologique ce qui ne permet pas de les apprécier à leur juste valeur (BENDALA GALÁn 199181 situe la propagation de ce type de tombe aux IIè et IIIè siècles ap. J.C.).

\section{L'inhumation et le mobilier funéraire}

La pratique d'entreposer des objets dans les tombes n'est pas propre au rite de l'inhumation (PRIEUR 1986 27; TURCAN 1958 323-347; TRANOY 2002 109). L'une des deux sépultures de Capera contenait, tout près de l'urne, des objets tels que des récipients en verre, notamment des coupes et des lacrymoires, une belle patère et deux cuillères d'argent, deux petits chaudrons, un couteau en fer. Selon A. Floriano, ce mobilier serait à mettre en lien avec la fonction sacerdotale du défunt (FLORIANO 1944 285).

A Las Merchanas, certains corps ont pu être déposés dans des cercueils de bois; on a retrouvé quelques clous: le bois aurait totalement disparu. D'autres corps étaient enterrés sans autre protection que des dalles de pierre (tombes à lauzes) ou des tuiles plates qui entouraient la 
fosse, à la surface. Certaines tuiles étaient peut-être disposées "en batière", des tuiles rondes (imbrices) faisant office de toit. La plupart des sépultures sont alignées, la tête vers le mur du castro. Dans les derniers rangs, cette disposition se brouille, certaines tombes se superposant. Aucune stèle n'a été mise au jour, ce qui donne une première indication chronologique: la nécropole ne serait pas antérieure au IIIè siècle ap. J.C. (MALUQUER 1968 119). Une grande partie des restes osseux découverts seraient, selon J. Maluquer, ceux d'enfants et d'adolescents. Le mobilier funéraire mis au jour est des plus sommaires: des récipients de céramique et de verre, parfois des outils de travail, des clous, diverses pièces difficiles à identifier (une amulette en bronze?) (MALUQUER 1968 115ss). Une des tombes est apparue mieux pourvue que les autres: la sépulture n. ${ }^{\circ} 17$ a livré les ossements d'un individu assez jeune avec quelques outils qui gisaient près d'un poignard de type Simancas. Déterminer avec précision le milieu social du défunt est délicat: très certainement un milieu relativement aisé par rapport aux autres défunts, peut-être un militaire. J. Maluquer avance d'ailleurs l'hypothèse de l'existence à Las Merchanas d'un castro renfermant un contingent militaire chargé de surveiller la production d'étain de l'ouest salmantin (MALUQUER 1968119 et 125; SALINAS 1994 179-180).

A Yecla de Yeltes, la nécropole à inhumation, datée du IVè ou Vè siècle, a livré des sépultures et un mobilier comparables (à l'exception des outils) à ceux de Las Merchanas, en particulier le poignard de type Simancas, décoré d'après P. de Palol, de motifs à la fois indigènes et de tradition romaine (PALOL 1964 92-94). Nous ne pensons pas que la présence de ce poignard dans une des tombes soit l'indice de la fonction militaire du défunt mais plutôt le témoignage d'une relative prééminence sociale, tout comme la boucle de ceinturon retrouvée dans une des tombes de la nécropoles de Carpio Bernardo (Villagonzalo de Tormes, prov. de Salamanque) (MARTín VALLS 1982 195).

Tout près, à Calvarrasa de Abajo (Cabrerizos), la nécropole à inhumation se détache nettement des exemples précédents. Il s'agit d'une nécropole qui a livré, ce qui est rare, à la fois des stèles funéraires et des sépultures (une vingtaine) composées de dalles irrégulières enfoncées verticalement dans le sol et recouvertes d'ardoises, ainsi que des sépulcres monolithes (au moins quatre). La destruction de la nécropole avant d'y effectuer les fouilles nécessaires nous oblige à considérer la même chronologie que pour le noyau de peuplement voisin: entre le milieu du IIIè et la fin du IVè siècles (PIÑEL 1981 217-225). 
Le groupe des nécropoles à inhumation précédemment décrit semble se rattacher, par certains aspects (type de sépulture et de mobilier funéraire, différenciation sociale de quelques tombes, présence d'armes) à un ensemble plus vaste de nécropoles situées à proximité du Duero (Palol 1958 209-217; Palol 1966 33ss; MarTín Valls 1982 196-197). Même si l'idée d'un limes et d'une importante population militaire le long de ce fleuve doit être rejetée (LE Roux 1982 393-395; FuENTES DomínGUEZ, 1989), on peut admettre a priori que le nord de la Vettonnie ait été sensible aux mouvements des peuples barbares qui ont caractérisé les derniers siècles de l'Empire et que les habitants de castros tels que Yecla et Las Merchanas aient vu leur société se modifier (dans le sens d'une militarisation et de l'enrichissement d'un groupe dirigeant) et leurs fortifications se renforcer dans un contexte de danger plus ou moins direct (MALUQUER 1968 107-108; BALIL 1970 618-619). On peut en outre se demander si les nécropoles plus méridionales de Vettonnie ont subi une telle évolution (celle-ci restant hypothétique) ou si elles diffèrent nettement des nécropoles septentrionales, notamment en ce qui concernent les rites funéraires.

Nous possédons en Vettonnie centrale et méridionale plusieurs ensembles funéraires susceptibles de nous renseigner.

A Valverde del Fresno, dans le nord-ouest de la province de Cáceres, on a mis au jour une seule tombe composée de deux grandes et larges dalles d'ardoise qui formaient les parois latérales tandis qu'un parement de gravats fermait l'extrémité la moins large de la sépulture. Celle-ci était recouverte d'un morceau d'ardoise. Le mobilier comprenait plusieurs fragments d'une céramique estampillée (un plat en argile), des morceaux de verre ayant appartenu à une bouteille et un ensemble d'outils en fer en partie comparables à ceux retrouvés à Las Merchanas. Aucune arme n'a été découverte. La chronologie de la tombe demeure peu précise: IVè-Vè siècles (d'après des critères de céramique) (GARCía de Figuerola 1984-1985 371-375).

A Monroy, non loin de la villa de Los Términos, à 200 mètres de l'ermitage Santa Ana (sans doute une ancienne église wisigothique), a été mise au jour une petite nécropole rurale (MOLANo BRÍAs et alii 1991-1992 141-170). Sept inhumations ont pu être fouillées mais seulement deux d'entre elles ont été retrouvées intactes. La zone, particulièrement riche sur le plan archéologique, a également livré des stèles funéraires du Haut-Empire (MARTín Gil 1932; Pujol 1974 185-193). L'inhumation a consisté à enterrer les défunts dans des fosses rectan- 
gulaires dont les "murs" étaient composés de morceaux d'ardoise ou, mais c'est moins fréquent, de briques ou tuiles (parfois plantées verticalement dans le sol) recouvertes de dalles d'ardoise qui ont été retrouvées à proximité immédiate. Deux tombes consistaient seulement en fosses creusées dans la roche, sans parement d'aucune sorte (GONZÁLEZ CORDERo 1989 137). Certaines des sépultures contenaient un maigre mobilier (anneau, céramique, clous), des restes osseux. Une des tombes a livré les ossements de deux personnes (peut-être trois) de sexe féminin, sans doute liées sur le plan familial, tout comme les défunts des tombes $n .^{\circ} 2$ et 6 qui pouvaient être la mère et le fils, à en juger par le fait qu'ils "partageaient" un côté de leur tombe. Sur le plan chronologique, les sépultures de Santa Ana semblent s’insérer dans une séquence pouvant aller du IVè au VIIè siècles. Elles intègrent des éléments d'organisation rituelle qui s'enracinent dans une tradition romaine (localisation de la nécropole, situation du mobilier, orientation de la tombe) avec des intrusions d'influence wisigothique. Il est certain que toutes les tombes ne sont pas contemporaines, et il est même possible que quelques-unes soient paléochrétiennes. Nous nous situons dans un contexte de transition du monde romain au monde médiéval bien différent de celui du groupe des nécropoles du Duero et de celui des nécropoles typiquement wisigothiques de la Meseta.

Plus au sud, à six kilomètres au sud de Campolugar (à la limite des provinces de Cáceres et de Badajoz), une ferme a livré non seulement quelques stèles funéraires mais encore un ensemble de trente sépultures que l'on peut subdiviser ainsi:

- un sarcorphage de marbre;

- un sarcorphage de granite;

- une série de fosses anthropomorphes constituées de parois faites de morceaux d'ardoise, de plaques de céramique ou de briques de fabrication courante, le couvercle étant en ardoises;

- trois tombes de facture beaucoup plus grossière: le défunt était déposé dans une fosse sommairement creusée, recouvert de terre puis de cailloux de quartz;

- des sépultures d'enfants constituées de plaques de céramique rappelant la forme d'un berceau (CALLEJO SERRANO 1969-1970 36-51).

Le mobilier funéraire était composé de fragments de céramique (CERRILlo 1980 51-64), de bijoux en bronze (en particulier un bracelet et un anneau), de quelques outils de fer (notamment un couteau et un 
clou), et d'une monnaie (en bronze) datant de l'empereur Probus (277-282) (Álvarez Rojas et García-Hoz Rosales 198849 n. 20).

Cet ensemble de sépultures appartient à différentes séquences chronologiques. Il s'agit probablement d'une zone funéraire qui fut utilisée sur une très longue durée et peut-être dès le Ier siècle ap. J.C.. Les stèles funéraires retrouvées sur place devaient appartenir à des sépultures (sans doute à incinération) qui n’ont pas été retrouvées (AE 1967 207209 216). Une période d'inhumation (dans des tombes composées principalement de dalles d'ardoise c'est-à-dire la majorité des sépultures) aurait succédé à la pratique de l'incinération (aux alentours du IIIè siècle?) avant de céder la place à une troisième nécropole (Vè VIè siècles) à laquelle il faudrait rattacher les sarcophages (CALLEJO SERRANO 1969-1970 51). Si la chronologie générale semble se détacher de l'examen des divers types de sépulture, le cadre culturel dans lequel ont évolué les défunts des deux dernières nécropoles est des plus obscure: ni l'influence du christianisme ni celle de la présence wisigothique n’apparaît.

\section{Les Stèles Funéraires}

On ne peut guère parler d'une typologie variée des monuments funéraires dans la Vettonnie du Haut-Empire, sous-entendant par là l'existence de plusieurs groupes nettement différenciés de monuments funéraires: stèles, cupae, autels, plaques, mausolées, hypogées. La quasi totalité des monuments funéraires sont des stèles qui devaient faire partie de tombes, vraisemblablement à incinération, même si, en ce domaine, il reste une part d'incertitude. Quelques cupae ont été identifiées à Ávila, de même quelques belles plaques comme celle de $M$. [...]ssius Silvanus à Salamanque ou celle qui se situe dans l'entrée du Palacio de Anaya, près de la cathédrale de cette même ville. Il existe également de rares dédicaces mais dont on doit nier le caractère funéraire (par exemple CIL II 834: dédicace en l'honneur de Bolosea, effectuée par M. Fidius Macer, à Capera: l'inscription provient de l'arc); à l'exception des verracos portant une inscription funéraire, aucun autre type de document n'est attesté. La classification qui peut être menée se fera donc principalement à l'intérieur de l'ensemble des stèles (auxquels on joindra le groupe des plaques). Celles-ci, sans présenter une grande variété, ne peuvent cependant être étudiées comme faisant par- 
tie d'un ensemble homogène. Des critères, non pas liés à la nature du matériau, mais à la forme, et plus encore à la décoration, interviennent dans cette classification.

Les stèles et plaques de facture "classique” (SALINAS 1995 282; АВÁSOLO et MARCO 1995 330-331)

Quelques stèles funéraires présentent un aspect qui tend à les rapprocher des stèles que l'on trouve dans les contextes les plus romanisés de la péninsule. Nous avons déjà évoqué l'inscription en marbre de $M$. [...] ssius Silvanus à Salmantica. On pourrait également évoquer la plaque funéraire en marbre retrouvée à Alcolea de Tajo (prov. de Tolède). De tels documents proviennent incontestablement d'un milieu favorisé et ouvert sur la romanité, peut-être un milieu d'origine italienne.

Mais ce type de stèle, non seulement est en nombre très restreint (même en considérant des documents perdus mais dont le texte suggère que le support de l'inscription devait être soigné), mais encore, aucun d'entre eux ne soutient la comparaison avec les stèles de caractère "classique" (de la Meseta nord) décrites par J. A. Abásolo et F. Marco (АВÁsolo et MARCO 1995 330-331). Ainsi, aucun monument en forme de maison ni même présentant un médaillon (clipeus) contenant la tête ou le buste du défunt n'a été recensé en Vettonnie (GARCÍA GELABERT et BLÁZQUEZ 1994 309-321). Mais les décors anthropomorphes n'en sont pas pour autant absents.

\section{Les stèles "à décor anthropomorphe” (Figure I)}

Les stèles funéraires de Torrejón el Rubio, de Talaván (prov. de Cáceres) et de Valdunciel (prov. de Salamanque) présentent ainsi une forme exceptionnelle dans le cadre vetton: il s'agit d'une forme simplifiée de la "stèle édicule ou pseudo-édicule" (GARCíA Y BELLIDO 1967 116-117). Dans les trois cas on a gravé un buste togé à l'intérieur d'une simple niche. Les trois personnages sont de face mais leur sculpture est encore rudimentaire. Le buste de Talaván a les mains croisées sur la poitrine. La stèle de Torrejón el Rubio pourrait dater du début du Ier siècle ap. J.C., à en juger par le type d'épitaphe (absence de référence aux Mânes) et serait la plus ancienne des trois (HEP 11989 186; GAMALLO et 
GIMENO 1990 283). Ces trois stèles sont incontestablement celles d'indigènes copiant plus ou moins maladroitement des modèles romains.

D'autres stèles vettonnes, toutes provenant de la province de Cáceres, présentent ou présenteraient des motifs anthropomorphes mais certaines ont été perdues si bien qu'il est difficile de confirmer les descriptions détaillées des historiens locaux. Un document retrouvé dans une ferme près de Plasenzuela comporte de façon incontestable une figure humaine dans son intégralité, debout, les bras en l'air, les mains ouvertes, les traits du visage étant grossièrement dessinés. Il s'agit d'un personnage féminin, sans doute un enfant qui figure sur toute la partie centrale de la stèle, la partie supérieure étant occupée par une épitaphe peu lisible (CALLejo 1967 112-113 n. 19). Les stèles de Belvís de Monroy et de Zarza de Granadilla présenteraient quant à elles des reliefs de couples (Cil II 5349; HurTAdo 197975 n. ${ }^{\circ} 80$ et 274 n. ${ }^{\circ}$ 662). A Zorita, la partie supérieure d'une autre stèle était occupée par "une tête humaine en haut-relief et une inscription illisible" (RAMÓN Y FERNÁNDEZ 1951 n. $\left.{ }^{\circ} 19\right)$. Beaucoup plus problématique est le cas du buste féminin en marbre représentant une scène d'allaitement qui se trouverait actuellement dans l'église de Jarandilla. En admettant son origine romaine (CALlEJo 1963 222-223), on serait alors en présence d'un monument funéraire particulièrement soigné et atypique en Vettonnie, proche par son aspect de certaines stèles de Mérida (NogALES 1994 201-210; Nogales 1997; EdmOnson, Nogales et TriLlmich, sous presse). Mais le monument en question donne une très nette impression de baroque: la stèle romaine aurait-elle été "remaniée", voire recopiée, au XVIIè siècle, date à laquelle elle a été publiée pour la première fois?

\section{Les stèles "à sommet astral"}

L'immense majorité des stèles vettonne appartient à un groupe très répandu dans la partie septentrionale de la péninsule ibérique. Il s'agit de stèles de forme rectangulaire ou même trapézoïdale (la largeur diminuant vers le bas) et dont le sommet est de forme arrondie. La partie supérieure de la stèle est occupée par un symbole astral, tandis que l'épitaphe se trouve en général dans la zone centrale, la partie inférieure étant parfois occupée par d'autres motifs (SALINAS 1995 283). A l'intérieur de ce groupe, il existe en fait une certaine variété de modèles qui témoignent vraisemblablement de l'existence de nombreux ateliers vet- 
tons car il est imprudent de supposer que de telles stèles proviennent toutes d'un seul centre de fabrication ou d'ateliers localisés dans le pays asture tout proche où ce type de monument funéraire, avec des variantes, est très représenté. Ainsi, de l'autre côté du Duero, dans la région de Miranda do Douro, au Portugal, se trouve le groupe des stèles dites de type "Picote" qui se caractérisent par leur décor astral et d'animaux (sangliers, taureaux); cependant, on constate la quasi-absence de motifs animaliers sur les stèles vettonnes, ces dernières se rapprochant davantage de celles de la vallée de l'Aliste où les stèles ne comportent presque plus de motifs animaliers (TRANOY 1981 350). En Vettonnie, on retrouve essentiellement deux grands types de stèles "à sommet astral" qui témoignent, au-delà d'un même fonds culturel, de différences artistiques liées aux particularités humaines des différentes zones de notre territoire:

- Le groupe ouest-salmantin, principalement localisé dans les nécropoles de Yecla de Yeltes et d'Hinojosa de Duero, dans une zone particulièrement riche en castros et qui présente une spécificité culturelle remarquée depuis longtemps, comprend des stèles assez uniformes: décoration astrale dans la partie supérieure, puis inscription. La partie inférieure est fréquemment décorée de motifs géométriques mais les figures humaines ou animales sont très peu représentées (MALUQUER 1956 36; NAVASCUÉS 1963 159-223; LOZANO 1973 105-108 et 113-114; MARTín VALls 1982 182-191) (Figure II).

- Le groupe d'Estrémadure se présente sous un aspect moins riche. Si on y retrouve les thèmes astraux (avec une plus grande fréquence du croissant lunaire que dans le groupe ouest-salmantin), les éléments qui constituent la décoration sont des plus simples et chaque stèle n'en comporte souvent qu'un seul. Ce groupe de stèles ne présente, la plupart du temps, aucune décoration dans la partie inférieure. De plus, un motif présent dans le groupe précédent semble absent en Estrémadure: l'arc (LOZANO 1973 107) (Figure III).

S'il est possible de discerner deux grands groupes de stèles "à sommet astral", il n'est guère raisonnable de les opposer: l'appartenance à un même ensemble culturel ne fait aucun doute. La plus grande simplicité des stèles d'Estrémadure s'explique-t-elle par une tradition indigène affaiblie (LOZANO 1973 114)? Il faut se garder de toute généralisation abusive: acculturation et tradition indigène peuvent coexister au sein d'un même site. Ainsi, à Yecla, quelques stèles funéraires ne présentent, à leur sommet, aucun motif astral (MARTín VALLS 1982 $189-190$ n. ${ }^{\circ} 11$ et 12$)$. 


\section{Les stèles avilaises}

Cet ensemble de stèles se différencie nettement des deux groupes précédents. Il s'agit de pierres tombales en granite que l'on pourrait être tenté de qualifier de purement indigènes en raison de leur facture extrêmement rudimentaire. Ces stèles se présentent sous la forme de blocs mal taillés, rectangulaires, avec la partie supérieure plus ou moins arrondie. Une majorité de ces stèles comporte de vagues motifs anthropomorphes, des portaits très idéalisés, parfois accompagnés de symboles astraux. Ces portraits sont réalisés sans le moindre souci de réalisme: de simples lignes permettent d'identifier des têtes, la plupart du temps sans dessin d'oreilles, de simples points faisant office d'yeux, de narines et de bouche (lorsque ces éléments du visage ont été gravés). L'étude de l'onomastique confirme le caractère indigène de l'épigraphie funéraire avilaise (RoDRíGUEZ ALMEIDA 1980 89-90; KNAPP 1992 10-72; HeRnANDO SOBRINo 1999 536-537). On retrouve ces caractéristiques plus au nord dans la péninsule, en Navarre, dans les provinces d'Álava et de Pontevedra (MARCo Simón 1978 43-44). On a d'abord effectivement considéré cette statuaire comme indigène "par essence" mais on doit également mettre en avant le fait que la nature du matériau utilisé à Ávila (granite) peut expliquer la simplicité des figures représentées (HERNANDO SoBRINO 1999 536-537). On doit enfin rappeler que nous nous situons là dans une des régions du conventus emeritensis les plus éloignées de Mérida d'où se sont propagés les modèles des portraits funéraires.

Une telle répartition en trois grands groupes est critiquable dans le sens où certaines stèles vettonnes n'entrent pas dans un tel classement, d'autres présentant un caractère mixte qui en font à la fois des stèles "indigènes" et des stèles présentant des influences venues de Rome, sans doute par le biais d'Emerita Augusta (sur l'ambiguïté de l'expression "stèle indigène" cf. ABÁsOlo et MARCO 1995 331ss). De plus, nous disposons de trop peu d'indications chronologiques pour affirmer que ces trois groupes ont été contemporains. On ne peut se fonder sur un tel classement pour diviser la société vettonne du Haut-Empire en trois grands ensembles. 


\section{Le symbolisme funéraire}

Essayer d'entr'apercevoir la conception qu'avaient les Vettons de la mort nous apparaît comme une démarche nécessaire pour mieux comprendre les motifs symboliques représentés sur les stèles funéraires.

Entr'apercevoir est bien le verbe qui convient car nous ne possédons malheureusement que très peu d'informations à ce sujet, les sources littéraires étant muettes. Deux données fondamentales doivent être cependant prises en considération. En tant que population d'origine indo-européenne et celtisée, les Vettons ont dû avoir des croyances en partie communes à celles de peuples voisins sur lesquels nous possédons davantage d'informations, notamment les Celtibères, voisins du nord-est, et les Astures, voisins du nord-ouest (TRANOY 1981 340-361; MARCo 1985 47-69; SopeÑa 1987; MARCO 1987 55-74). Une telle démarche est d'autant plus justifiée que nombre de motifs présents sur les stèles funéraires astures et celtibères se retrouvent sur les stèles vettonnes (MARCO 1978; ACUÑA 1974 17-31; MARCO 1976; RODRÍGUEZ LAGE 1974; Tranoy 1981 349-350). De plus, tout comme les Vettons ont continué, sous domination romaine, à adorer des divinités protohistoriques, une grande partie de leurs croyances sur l'au-delà a dû perdurer. La référence à des sources préromaines se justifie donc.

Certaines divinités étaient sans doute davantage liées au monde des morts que d'autres: Endovellicus, Ataecina sont souvent considérés comme des dieux funéraires (SÁNCHEZ Moreno 1997 118-120).

Les astres devaient être revêtus d'une valeur funéraire bien qu'il soit difficile de préciser si le soleil, la lune, les étoiles, représentés sur certaines stèles, étaient considérés comme des dieux, comme des demeures des dieux (et des morts?) ou comme des téophanies. Comme nous le verrons, leur relation avec le monde des morts ne semble pas faire le moindre doute et cette relation n'est d'ailleurs pas propre aux Vettons (ABÁsolo et alii 1975 67-68).

La croyance selon laquelle les défunts atteignaient une autre vie, croyance généralisée dans la péninsule ibérique, avant et après l'arrivée des Romains, est confirmée, en Vettonnie, par la présence de mobilier funéraire à proximité immédiate des restes du défunt, incinéré ou inhumé. L'absence de mobilier funéraire s'explique davantage par les destructions et pillages ou par le milieu social défavorisé du défunt, en aucun cas par l'incroyance en la survie du défunt dans l'au-delà (MANGAS 1978 608-611; MORRIS 1992). 
Dieux infernaux et pratiques magiques devaient également faire partie des croyances funéraires des Vettons. Dans les nécropoles protohistoriques de Vettonnie, la relation étroite qui semble s'être instaurée entre le guerrier vetton et son arme, l'évocation de Strabon concernant un Vetton pour qui seuls la guerre et le repos étaient envisageables relèvent peut-être d'une mentalité magique qui n'a pas dû complètement disparaître à l'époque romaine (STRABON III 4 16). Les fourreaux des épées de Las Cogotas et de La Osera sont décorées d'amulettes circulaires, motifs que l'on retrouve sur les céramiques de ces mêmes nécropoles ainsi que dans celle de Numance (CABRÉ 1953 101ss; GRAF 1994). Selon J. M. Blázquez, le ceinturon retrouvé dans la sépulture n. ${ }^{\circ} 350$ de la nécropole de La Osera avait une valeur magique (BLÁzQUEZ 1985 150). Cette mentalité a perduré sous la domination romaine, en se transformant, car le monde de la guerre a perdu de son importance sous la domination romaine. Près de l'arc de Capera, on a retouvé une représentation de phallus qui témoignerait de la survivance de la magie à l'époque romaine (BLÁZquez 1965a 34 et pl. X, 3; BLÁZQUEZ, 1985 149).

L'utilisation des verracos à des fins funéraires témoigne de la survivance et de la transformation de pratiques magiques protohistoriques dans un contexte funéraire. Nous essayerons de mieux cerner la relation entre ces sculptures zoomorphes et le monde des morts.

En revanche, l'exposition des morts, pratiquée dans le nord de la péninsule, ne semble pas avoir existé dans la Vettonnie romaine (MARCo Simón 1993 502; SÁnCheZ Moreno 1997 128-129).

Les motifs astraux sont les éléments du symbolisme funéraire les plus courants en Vettonnie.

Dans la province de Salamanque, le motif le plus répandu est celui de la roue dite solaire. Il s'agit d'une circonférence comportant des rayons internes de formes diverses et qui est toujours située, lorsqu'elle est présente, dans la partie supérieure de la stèle (NAVASCUÉS 1963 159-223; CALLEjo 1977 152, qui évoque un cercle au pied de quelques rares stèles de la province de Cáceres). Les rayons sont presque toujours courbés, tournés vers la droite ou vers la gauche, mais quelques-uns sont en forme d'hélices, de pétales, de croix, de bâtonnets extérieurs, voire de simples trous comme sur une stèle d'Ávila (RoDRíGuEZ AlmeIDA 1980 141-142 n. ${ }^{\circ}$ 56). Leur nombre ne dépasse pas vingt et on constate aisément que ce sont les disques solaires à 6 rayons qui sont les plus nombreux, avec une légère prédominance des rayons décentrés 
vers la droite. Certains cercles comportent des pétales intérieurs (6 ou 4): ces motifs, assez nombreux dans la province de Cáceres, sont souvent appelés rosaces à pétales; certains auteurs les distinguent des rouelles solaires (CALLEJO SERRANO 1977 153-154). Il n'a pas été possible de mettre en relation tel type de rayon avec un groupe humain ou social bien précis: les stèles funéraires des femmes présentent des disques solaires à rayons aussi bien tournés vers la droite que vers la gauche; les quelques rouelles solaires à rayons droits, en croix ou en hélice ornent les stèles d'individus ordinaires. Le fait que la stèle d'Aprodite (vraisemblablement une esclave) soit décorée d'un disque à rayons en hélice n'a sans doute aucun lien avec sa condition servile (NAVASCUÉs $200 \mathrm{n} .^{\circ} 53$ ). Nous ignorons en fait si la forme des rayons (y compris les pétales) avait une signification particulière.

Dans la province de Cáceres, à Monroy, une stèle "à sommet astral" présente une configuration sans parallèle dans toute la Vettonnie: le soleil est représenté de façon relativement réaliste, avec un large cercle (45 cm de diamètre), dont la circonférence a été tracée au compas (le centre du cercle est facilement identifiable), et d'où partent un nombre important de petits rayons. C'est semble-t-il le seul cas dans toute la Vettonnie où les rayons sont à l'extérieur du cercle. Nous ignorons si le nombre de rayons est en relation avec l'âge,non précisé par ailleurs, du défunt, Suriacus Turanif. (PuJol 1974 185-193) (Figure IV).

Il est extrêmement délicat de chercher à éclaircir la signification de ces rouelles solaires. En premier lieu, nous ignorons si les rosaces à pétales doivent être considérés ou non comme des soleils. On peut également douter de l'origine précise de ces motifs car le thème du soleil, symbole d'éternité, de vie et de fécondité, lié à l'au-delà, n'est pas propre au monde celtique (CuMONT 1981 61). Ainsi, l'hypothèse d'une origine orientale, d'une pénétration de ce thème par le biais de l'occupant romain serait totalement acceptable si le thème solaire ne s'enracinait en fait dans un fonds protohistorique indigène. Tout comme l'importance des astres est attestée dans la religion celtique, dans et hors de la péninsule, de nombreuses représentations du disque solaire ont été retrouvées dans toute la Meseta (BLÁzQUEZ 1975 119-120). A Las Cogotas et à La Osera, des figures anthropomorphes du soleil ont été identifiées (MANGAS 1978 596). A Las Cogotas, ce motif était représenté sur une barque, ce qui tenderait à renforcer le lien du soleil, symbole d'immortalité, source de vie, avec le monde de l'au-delà. Cependant, dans le cas de la stèle de Monroy, on peut se demander s'il 
ne s'agit pas d'une influence orientale dont porterait par ailleurs témoignage le nom du dédicant: Suriacus (ABASCAL 1994 518). Cependant, on remarquera que le père de Suriacus porte un nom indigène: Turanus.

Sous forme de croissant, la lune apparaît également, seule ou accompagnée d'autres motifs astraux, sur de nombreuses stèles funéraires, tout particulièrement dans la province de Cáceres, mais aussi, dans une moindre mesure cependant, à Ávila (RoDRíGUEZ ALMEIDA 1980 90). On ne connaît en effet, dans la province de Salamanque que deux documents sur lesquels soit représenté avec certitude un croissant lunaire, en position horizontale, les pointes vers le haut. Il s'agit tout d'abord de la stèle d'Arcoturus Pisirif. (décédé à 15 jours) à Puebla de Azaba, dont le milieu du texte est interrompu par divers motifs, dont un croissant lunaire. L'autre document est la stèle érigée par le centurio Reburrus à sa mère Bolosea (Figure V). Dans ce dernier cas, la lune occupe le haut de la stèle, comme sur presque toutes les autres stèles vettonnes où le croissant lunaire est représenté (CALLEJO 1977151 cite deux inscriptions où la lune est située dans la partie inférieure de la stèle). C. Callejo Serrano a répertorié onze différents types de croissants lunaires en Haute-Estrémadure et a noté que le croissant comporte parfois des détails tels que des traits rattachés à la partie convexe du motif, des appendices terminés en cercles, etc... détails dont la signification - s'il y en a une - demeure obscure (CALLEJo SERRANO 1977 149-151). La présence même des croissants lunaires sur les stèles est loin d'être claire. Nous savons que la lune était l'objet d'un culte parmi certaines populations de la péninsule, en particulier les Callaïques, les Celtibères et les Vaccéens (STRABON III 4 16; APPIEN Iber. LXXXII). L'hypothèse la plus aisée à avancer pour expliquer sa présence sur les stèles funéraires est de considérer cette représentation comme la demeure des morts, peut-être en raison du caractère lumineux de la lune nocturne. La diffusion du croissant lunaire en Vettonnie doit-il être mis en relation avec l'influence des religions orientales? On remarquera en premier lieu que l'onomastique indigène prédomine largement sur les stèles présentant ces lunules et que c'est en Haute-Estrémadure, zone montagneuse très empreinte de traditions indigènes, que l'on trouve la plus grande concentration de lunes. Même en considérant la théorie de J. J. Hatt (concernant le symbolisme astral sur les stèles funéraires gauloises), qui avance l'idée d'un syncrétisme celto-oriental "sur la frange d'interférence entre la propagande mystique gréco-orientale et le fonds indi- 
gène”, nous ne croyons pas, à une influence orientale, ou alors de manière très ponctuelle (Monroy), sur les stèles vettonnes (HATT 1981 387-388; Cumont 1942 177-252; Kooy 1981 45-62).

D'autres éléments, en proportion très nettement inférieure aux rouelles et lunules, sont présents sur quelques stèles vettonnes, en particulier les arches et les équerres.

Certaines stèles funéraires, en particulier dans la province de Salamanque, sont ornées, sous l'épitaphe, de larges canelures en forme de rectangles ou plutôt d'arches car la plupart du temps, ces motifs sont voûtés (Figure VI). Sur un document particulièrement endommagé provenant de Yecla de Yeltes, les canelures se terminent par une véritable tête, de forme arrondie, nettement détachée du reste du motif, le tout prenant l'apparence d'une quille (NAVASCUÉs 1963 219-221 n. ${ }^{\circ}$ IX). Le nombre de ces arches est généralement de deux ou trois par stèle, parfois quatre comme sur la stèle déjà évoquée de Puebla de Azaba dont les arches consistent en quatre rectangles creusés dans la pierre (comme tous les motifs des stèles funéraires) et, représentation unique dans toute la Vettonnie, reliés entre eux par la base et encadrés par ce qui ressemblerait à une maison (la demeure des morts?) (Figure V). Que symbolisent ces arches? S'agit-il de figurations des portes de l'au-delà, c'est-à-dire les portes qui, dans la mythologie gréco-romaine (et peut-être celtique?), permettent de pénétrer dans la demeure du dieu des Enfers, comme le pense notamment J. M. Blázquez (BLÁzQUEz 1965a 14)?

Quelques inscriptions funéraires sont décorées d'une paire d'équerres ou d'une double équerre (moins répandue), localisées un peu au-dessous du disque solaire, de chaque côté de la stèle. La paire d'équerres présente presque toujours le bras vertical vers le haut et le bras horizontal vers le centre de la stèle. La double équerre, quant à elle, se trouve située au même endroit mais le bras horizontal est commun et relie les deux bras verticaux. Ces équerres peuvent présenter des formes légèrement différentes: certaines sont larges et se terminent par de petites pointes concaves, d'autres consistent en de profondes et fines incisions dans la pierre. Sur une stèle d'Hinojosa de Duero, deux paires d'équerres, en haut et en bas de la pierre, orientent leurs bras verticaux vers le pied (NAVASCUÉs 1963171 et n. ${ }^{\circ}$ LIV). Ces équerres devaient être imprégnées d'une valeur religieuse - du moins à l'origine - car leur aire de dispersion dans la péninsule est trop étendue pour qu'il puisse seulement s'agir d'un motif décoratif (TURCAN 1978 1701-1702). Si F. Cumont n'a pu éclaircir la signification de ces équerres, d'autres auteurs 
ont écrit qu'il pourrait s'agir des bras d'Atlante supportant la voûte céleste, interprétation des plus incertaines (CUMONT 1942 229ss; BLÁZQUEZ 1983 270).

D'autres éléments apparaissent sur les inscriptions vettonnes: quelques feuilles de lierre, symboles d'immortalité, de rares swasticas, des torques (héroïsation du défunt?) (NAVASCUÉs 1963172 et n. ${ }^{\circ}$ LXIII: stèle d'Acinicia à Yecla); le thème animalier se limite, chose surprenante, à un seul document: la stèle d'Apana Triti et Apana Clouti, retrouvée à Villar de la Yegua (province de Salamanque), dont la partie inférieure était ornée de la figure d'une biche, à moins qu'il ne s'agisse d'une chèvre ( $c f$. Figure V). Le culte de la biche, bien connu en pays lusitanien, est donc à peine perceptible en territoire vetton, où cet animal a pu être revêtu, du moins chez certains individus (d'origine lusitanienne?) d'une valeur funéraire (Aulu-Gelle XV 22; Plutarque Sert. XI 2; APPIEN Iber. C). Une telle signification est attestée dans d'autres zones celtisées de la péninsule, notamment dans le nord-ouest (TRANOY 1981 236).

\section{Les épitaphes}

La partie essentielle des stèles funéraires est le champ épigraphique qui porte les épitaphes. Leur position centrale, l'espace occupé par l'inscription sont des indices révélateurs de l'importance qu'on accordait à l'épitaphe au moment de la confection de la stèle. Aucune stèle ne semble avoir totalement négligé ou escamoté, ce qui est compréhensible, l'épitaphe au profit d'un autre type d'information, par exemple des figures symboliques ou de pure décoration; ces dernières apparaissent toujours comme des éléments secondaires de la stèle. Les textes funéraires nous fournissent l'essentiel des informations relatives au milieu social, voire familial du défunt, notamment les liens de parenté, l'organisation supra-familiale, quelques éléments relevant de la démographie, les mouvements de population, les relations maître/esclave (BonNAud 1999 III 29-105). De même il a été possible, à partir des nomina gentilicium de Lusitanie, de parvenir à une réflexion non dénuée d'intérêt sur l'émigration italique en Lusitanie (NAVARRo CABALLERO 2000 281-297) bien que la Vettonnie apparaisse singulièrement comme une zone où l'immigration a été faible. Notre propos n'est pas de reprendre ces informations mais de considérer les épitaphes comme 
un élément essentiel de la stèle et de brièvement rappeler les différents éléments de ces textes funéraires.

\section{La formule indigène}

Les épitaphes présentent en effet un modèle d'organisation relativement simple, et ce malgré l'existence de différents types de stèles. Ces derniers ne se distinguent donc nullement par la structure de leurs épitaphes qui correspond à un formulaire utilisé bien au-delà du pays vetton et que nous rappelons brièvement: l'inscription débute souvent par l'invocation aux Mânes, toujours exprimés sous une forme abrégée; cette invocation n'apparaît cependant pas toujours sur les stèles vettonnes; ensuite vient le nom du défunt, au nominatif (Ier siècle ap.J.C.) ou au datif (stèles plus tardives), dont la structure et l'origine dépendent du contexte culturel et du degré d'intégration au monde romain; puis l'âge, rarement précédé de vixit enfin une formule finale: hic situs (-a) est sit tibi terra levis, exprimé en abrégé de différentes façons. Ainsi, en Vettonnie, la plupart des stèles semblent combiner en une seule les deux formules $h(i c) s$ (itus) e(st) et $s($ it) t(ibi) t(erra) l(evis). Les abréviations sont parfois incomplètes: h.s.t.t.l., h.s.t.l., s.t.t.l....(sur ces formules $c f$. BRELICH 1937 10ss). On peut dire que, sauf exception, toutes les épitaphes de Vettonnie se présentent sous cet aspect, souvent qualifié d'indigène, dépassant rarement les cinq lignes.

A ce schéma se rajoute souvent la filiation du défunt, sa tribu s'il est citoyen romain (mais la plupart des tria nomina ne sont accompagnés d'aucune mention de tribu), éventuellement la structure supra familiale indigène. Enfin, le ou les dédicants apparaissent parfois dans l'inscription. Au-delà de ces ajouts éventuels, certaines inscriptions se démarquent plus radicalement de la structure précédemment décrite.

L'invocation aux Mânes (BRELICH 1931 24ss; GORDON 198340 ss; CORBIER 1998 25)

Elle se fait généralement sous la forme $D$ (is) M(anibus) S(acrum), éventuellement sous la forme $D . M$, exceptionnellement $D$ (is) $M$ (anibus) I(nfernis) ou D(is) Manibus (MALUQUER 1956 n. ${ }^{\circ} 66=$ HAE 1291; CIL II 5312 = MORÁN 1922 n..$^{\circ}$ 158; CIL II 875 = MORÁN 1922 n. $.^{\circ} 219=$ MALuQuer 1956 n. ${ }^{\circ}$ 102). Dans la province de Salamanque, la consécration aux Mânes n'est cependant pas majoritaire (NAVASCUÉS 1963 173); certains sites, comme celui de Yecla sont peu attachés à cette pra- 
tique, avec environ un quart des inscriptions qui comportent cette invocation aux Mânes. En revanche, à Hinojosa, les découvertes les plus récentes témoignent d'une référence aux Mânes beaucoup plus systématique, ce qui n'empêche pas la présence de symboles astraux sur les mêmes stèles (JIMÉNEZ DE FURUNDANERA 1993 133-160; HERNÁNDEZ GUERRA et alii 1994 317-319). On notera également la particularité des épitaphes de ce site où prédomine la formule h.s.t.t.l. au lieu de h.s.e.t.t.l. et où une inscription commencerait par posuit. Toujours dans la province de Salamanque, si on considère les épitaphes des stèles retrouvées dans des sites où la romanisation semble avoir été plus intense, cette faible proportion varie peu; et l'abréviation D.M.S. n'est d'ailleurs pas obligatoirement accompagnée d'une onomastique romanisée. Dans la province d'Ávila, la consécration aux Mânes est encore moins courante avec seulement huit documents épigraphiques (KNAPP 1992 358, tableau 18). R. C. Knapp a par ailleurs remarqué que presque toutes ces invocations aux Mânes sont gravées sur des verracos, comme si la tradition indigène, à la fin du IIè ou au début du IIIè siècle, avait récupéré la tradition romaine d'invoquer les Mânes (KNAPP 1984 219-230; KNAPP 1992 358). Dans la province de Cáceres, la mention des Mânes est également loin d'être prédominante. Dans la région de Turgalium, la plus ouverte sur l'influence romaine de toute la Vettonnie (REDONDO RODRÍGUEZ 1987), la proportion des stèles comportant l'invocation en question est si faible qu'on ne peut considérer cette dernière comme un indice de romanisation. Ainsi, il apparaît que l'invocation aux Mânes n'est pas à mettre en relation avec un milieu romanisé; mais la relier, sans nuance, au milieu indigène est tout aussi abusif. En fait, il semble bien que la consécration aux Mânes est à situer dans un contexte chronologique relativement précis (fin IIè-IIIè siècles) qui pourrait correspondre, à en juger par les exemples d'Ávila et d'Hinojosa, à l'existence de pratiques indigènes sous une façade romaine (LAMBRINO 1965 223-242; ETIENnE 1973 163). En ce sens, l'évocation des Mânes sur les stèles et verracos vettons peut difficilement être interprétée comme ayant une réelle valeur religieuse (LAMBRINO 1965 241).

\section{Défunts et dédicants}

Nombre de stèles funéraires comportent le nom d'un ou plusieurs dédicants, la plupart du temps un membre proche de la famille (BON- 
NAUD 1999 III 29-35). La formule $f($ aciendum) $c$ (uravit) peut alors apparaître à la fin de l'épitaphe comme sur cette inscription de Salamanque: D.M.S./C. Iulio Narcisso ann/XXI Iulia Thetis Mater f.c. (CIL II 877; MORÁn 1922 126; MALUQUER 1956 n. ${ }^{\circ}$ 26); quelquefois même avant h.s.e.s.t.t.l.: D.M.S./Apana Triti an./LX Apana Clo/uti f. an.V/ /Amaenea Ma/tri et filiae f.c./h.s.e.s.t.t.l. (AE 1983 503; MARTín VALLS 1982a 214-216, Villar de la Yegua, prov. de Salamanque); parfois f.c. est remplacé par d'autres expressions, beaucoup plus rares cependant, telles que posuit, curavit, $p$ (oniendum) c(uravit), statuit, sibi, sibi fecit: Quintus Modesti f. a(nnorum) XXV/Placida Modesti f. a(nnorum) XIII/Boudica Flacci f. Modestus/Celtiatis f. liberis uxori sibi feci[t?] (AE 1988 697; CIL II 455). On remarquera, dans cette dernière inscription, retrouvée à São Estevão (Sabugal, Portugal), l'abréviation peu courante de a(nnorum), au lieu de an(norum) ou à la rigueur ann(orum) (STYLOw 1995 222). Comme pour l'onomastique, des déformations orthographiques sont parfois perceptibles lorsque ces expressions ne sont pas abrégées, par exemple feicit au lieu de fecit à Vila Boa (Sabugal); l'inscription débuterait par Monim/entum (CURADO 1988 n. ${ }^{\circ}$ 123.1).

Des formules telles que ex testamento sont attestées en Vettonnie: Talaceo/Corporici (filio)/Cilius lib(ertus)/ex testament(o) (CURADO n. ${ }^{\circ}$ 123.2; AE 1989 389, à Vila Boa, Portugal). Dans ce cas précis, une telle expression renforce l'idée de l'étroitesse du lien patron/affranchi; ce dernier, plutôt qu'un membre de la famille étroite (ou nucléaire), a été désigné par testament pour faire ériger la stèle de son ancien maître. Néanmoins, dans la majorité des cas, la mention du dédicant n'est suivi d'aucune expression verbale, comme ici à Escalhao, Figueira de Castelo Rodrigo: Modestus Ambati f. Co/bel(cus) an(norum) LX Cornelia/Gensulia an(orum) $L$ h(ic) $s$ (iti) s(unt) $s($ it $) / v$ (obis) t(erra) l(evis) C(aius) Ammius Mode/stinus patri Firmus/Modesti lib(ertus) patro(no) (CIL II 433; HeP 21990 793). L'espace disponible sur la stèle entrait en ligne de compte. Ainsi, dans cette dernière inscription, les noms des dédicants, leur lien familial avec les défunts ont dû prédominer et peuvent expliquer l'absence de l'expression verbale finale.

Le nom du dédicant est parfois étrangement présent en tête de l'inscription. C'est le cas de la stèle funéraire de Malcenus, où le nom du défunt, Malceno Maelonis filio, n'arrive qu'en deuxième position dans le texte, sans indication d'âge, une fois mentionné le nom de son amicus: [Ca]esilius/[M]aelonis/[f(ilio)] amico/[su]o [statuit?] (AE 1990 520). Cette formulation peut traduire le caractère inégalitaire de 
l'amicitia unissant ces deux personnages (SERRANO Delgado 1987-88 345-364). Une telle présentation apparaît également sur la stèle de Puebla de Azaba (prov. de Salamanque), malheureusement fragmentée, et dont nous avons déjà évoqué les motifs symboliques qui interrompent l'épitaphe:

[...] facien/du(m) cura/vit/(motifs symboliques)/Arcotu/rus Pisi/ri f. XV d(ierum) (Figure V).

Dans la partie supérieure de la stèle devait être gravé le nom du dédicant. Outre l'âge du défunt - le seul dans toute la Vettonnie qui soit exprimé en nombre de jours, le $d$. de dierum étant placé après le chiffre (CRESPO et AlONSO 1999 b 95-119) - il faut remarquer le caractère particulier de ce document, unique dans toute l'épigraphie vettonne. L'âge du défunt explique sans doute la position de son nom après celui du dédicant (probablement les parents). Cet enfant, malgré son décès précoce, a "bénéficié" d'une stèle funéraire, ce qui est un cas unique dans toute la Vettonnie; nous pensons que ce fait doit être mis en relation avec le niveau social de ses parents qui ont tenu à ce que leur enfant ait sa propre stèle funéraire, sans doute davantage par souci de prestige social que par habitude religieuse, la pratique n'étant pas de faire ériger une stèle à un enfant prématurément disparu (sur les problèmes de l'étude de l'âge des décès $c f$. LASSÈrE 1987 91-97; SALMON 1987 99-112; HopKINS 1987 113-126).

L'affliction des familles des défunts n'apparaît pour ainsi dire pas sur les stèles vettonnes, ce qui ne confirme pas l'opinion de A. Balil soulignant au contraire les sentiments de parenté, d'amour et d'amitié qui sont continûment exprimés dans l'épigraphie hispanique (BALIL 1992 57). Cela peut s'expliquer par l'absence de tradition consistant à exprimer par écrit sa douleur et aussi par le caractère omniprésent de la mort dans ces sociétés.

Un document présente une épitaphe qui ne répond cependant pas du tout à cette norme. Il s'agit de la stèle funéraire retrouvée à Alcolea de Tajo (prov. de Tolède) (Figure VII):

Hordine. si. iusto placui/s. set. currere. fatis. hic. non/debuit. mori. an(norum). XX. set. potius/ante. pater. et. mater. debuerunt/es. se. sepulti. Infelices. mis(erum) tum/ulum. $f($ aciendum) c(uraverunt). parentes. Hic.erit. nuc/tumuli. dominus. ospitio/miseros. invita(t). parentes. L(ucius). Av(inius)/Tropimus. p(ater) et Luc. ceia. Vrbana. m(ater)./ L(ucio). Avinio. Avito. f(ilio)/pien. tis. simo. fa(ciendum) c(uraverunt). 
Nous proposons la traduction suivante:" S'il avait plu au destin de suivre son cours normal, il n'aurait pas dû mourir à vingt ans mais plutôt que son père et sa mère soient enterrés avant lui. Les malheureux parents ont pris soin de faire faire la sépulture pour le pauvre enfant. Désormais il sera le maître de la tombe et y convie ses malheureux parents. Lucius Avinius Tropimus, son père, et Lucceia Urbana, sa mère, ont pris soin de faire ériger ce monument à leur fils très pieux Lucius Avinius Avitus".

Ainsi, nous sommes en présence d'une épitaphe où l'idée de la mort prématurée d'un enfant est bien mise en exergue, ainsi que la douleur de ses parents qui ont pris soin de préciser qu'il s'agit d'une séparation momentanée (ospitio miseros invitat parentes). Qu'il s'agisse là d'une inscription émanant d'un milieu social aisé ne fait aucun doute: l'aspect extérieur du document (nature de la pierre: marbre, nombre important de lignes, décoration...) en est un premier indice, confirmé en cela par la longueur du texte et les tournures de phrases. Les fautes d'orthographe (hordine, set, nuc, ospitio, Tropimus) et le caractère superflu de plusieurs points ne doivent pas nous étonner dans la zone rurale où a été retrouvée l'inscription. Le système onomastique latin (tria nomina, origine latine de tous les noms) pourrait faire penser à une famille italienne même si ce n'est pas certain. Pour ce document, R. Martín Valls a proposé comme date le milieu du IIè siècle ap. J.C. (MARTín VALls 1971 429).

Enfin, on ne trouve nulle trace en Vettonnie d'uns stèle faite par et pour un individu de son vivant (se vivo sibi) (EDMONDSON 2000 309).

\section{Les viciniae}

De la cité de Capera, proviennent trois inscriptions qui se détachent également de l'ensemble des épitaphes vettonnes:

- [...]iulalex.inpens(a)/funeris/vicinialf(aciendum) c(uravit) (HABA et RoDRIGO 1986 44-46).

- Saluti/vicinia/Capere/nsis (CIL II 806).

- C.Cami/lius. Pa/ternus/Gal(eria tribu) Clu/nensis/an(norum) XXV/h.s.e.s.t./l. vicini/a. Clun/iesium/f(aciendum) c(uravit) (CIL II 821). 
La première de ces inscriptions est funéraire et a été découverte il y a une dizaine d'années à Villar de Plasencia; elle pourrait dater de la deuxième moitié du Ier siècle ap.J.C. (HABA et RoDRIGO 1986 46). Elle semble faire référence à un "voisinage" (vicinia) qui aurait pris en charge les dépenses des funérailles.

Le deuxième document est une dédicace à Salus, divinité aquatique adorée en d'autres lieux de Vettonnie. Les auteurs de cette dédicace sont également les membres d'une vicinia, cette dernière portant l'origo Caperensis. S'agit-il exactement de la même structure que dans le cas précédent? La présence de l'origo nous inciterait à répondre par la négative mais il s'agit d'une dédicace en l'honneur d'une divinité et non une inscription funéraire. On a pu vouloir préciser l'appellation complète de la vicinia pour rendre plus solennel le document.

Enfin, la troisième inscription est la stèle funéraire d'un homme originaire de Clunia et décédé à Capera. Il devait faire partie de la communauté des Clunienses implantés à Capera et dont l'épigraphie et la céramique nous ont livré quelques témoignages (AlvarADO 1994 100; GómEZ PANTOJA 1999 91-108). Le plus intéressant est de constater que c'est la vicinia Cluniensium qui a pris à sa charge les frais de la stèle funéraire et peut-être davantage. Il nous faut donc admettre l'existence à Capera d'une communauté de Clunienses assez organisée pour avoir engendré une structure capable d'assumer les frais funéraires de ses ressortissants.

Quelle réalité recouvrait précisément le terme vicinia? Qu'il s'agisse d'une organisation de type collégial (collegium), prenant à sa charge les frais funéraires, cela ne semble faire aucun doute. Néanmoins, ces viciniae avaient-elles une assise relativement précise, par exemple un quartier ou un vicus (vicinia Caperensis = vicinia du cheflieu de la cité)? Ou bien le sens premier de vicinia (voisinage) s'était-il élargi au point d'être l'expression sociale d'une communauté relativement précise (RodRíGUEZ NeILA 1976 10-118).

\section{Verracos et inscriptions funéraires}

L'étude des relations entre les Vettons et la mort ne serait pas complète sans évoquer la délicate question des verracos gravés d'une inscription funéraire (CERDEÑo et CABANES 1994 103-109; CASTELO et SÁNCHEZ Moreno 1995 317-330; Álvarez-SAnchís 1999 215-294; SÁNCHEZ 
MoRenO 2000 138-148, parmi les plus récentes publications sur les verracos). Il existe en effet en Vettonnie un petit nombre de sculptures zoomorphes qui portent des épitaphes, parfois sur les deux flancs. Rappelons que sur les 280 pièces recensées en son temps par G. López Monteagudo, seulement 25 portent des inscriptions, dont 18 proviennent du pays vetton, les autres se situant à proximité immédiate de la Vettonnie, en particulier en territoire asture (LÓPEZ MoNTEAGUdo 1989 125-138) (Figure VIII).

Tableau des inscriptions sur verracos (LÓPEZ MONTEAGUDO 1989 125-138)

Le numéro du catalogue correspond à celui de G. López Monteagudo.

\begin{tabular}{|c|c|c|c|c|c|c|c|}
\hline Mânes & Nom du défunt & Filiation & Age du décès & Dédicant & $\begin{array}{l}\text { Formules } \\
\text { funéraires }\end{array}$ & $\begin{array}{c}\text { Catalo } \\
\text { gue }\end{array}$ & Provenance \\
\hline Oui & Vari & & & & & 10 & Ávila \\
\hline Oui & $\begin{array}{c}\text { Cadano } \\
\text { Caburiq(um) }\end{array}$ & & & $\begin{array}{c}\text { Balarus } \\
\text { pat(er) }\end{array}$ & f.c. & 11 & Ávila \\
\hline \multirow[t]{2}{*}{ Oui } & Reb[urrus] & $\begin{array}{l}\text { Ma[gil]- } \\
\text { on[isf.] }\end{array}$ & & mat(er) & $\begin{array}{l}\text { h.s.e./ } \\
\text { s.t.t.l. }\end{array}$ & 12 & Ávila \\
\hline & Burro & $\begin{array}{l}\text { Magilo- } \\
\text { nis } f .\end{array}$ & & & & 27 & Ávila \\
\hline \multirow[t]{15}{*}{ Oui } & $\begin{array}{c}\text { Titillus } \\
{[\ldots]}\end{array}$ & & & Titullus & f.c.? & 56 & $\begin{array}{c}\text { Martiherrero } \\
\text { (AV) }\end{array}$ \\
\hline & Gaia? & & & & & 85 & $\begin{array}{c}\text { Guisando } \\
\text { (AV) }\end{array}$ \\
\hline & & & & $m a[t](e r)$ & f.c. & 87 & $\begin{array}{l}\text { Guisando } \\
\end{array}$ \\
\hline & $\begin{array}{c}\text { Longinus } \\
\text { Prisco } \\
\text { Calaetiq }(u-m)\end{array}$ & & & $\begin{array}{c}\text { (filius) } \\
\text { Textuelle- } \\
\text { ment : patri }\end{array}$ & f.c. & 88 & $\begin{array}{l}\text { Guisando } \\
\quad(c f . \\
\text { Figure IX) }\end{array}$ \\
\hline & Cauru[s...] & $M u[\ldots f] ?$. & $X X V I$ & & & 98 & $\begin{array}{c}\text { Tornadizos } \\
\text { (AV) }\end{array}$ \\
\hline & & & $X X$ & & & 105 & Tornadizos \\
\hline & Viviros? & & & & & 107 & Tornadizos \\
\hline & $\begin{array}{l}\text { Ateroe- } \\
\text { con? }\end{array}$ & & & & & 126 & $\begin{array}{c}\text { S. Jão } \\
\text { Pesqueira } \\
\text { (Beira Alta) }\end{array}$ \\
\hline & $\begin{array}{c}\text { Andalitia } \\
\text { mallgeinif. }\end{array}$ & & & $\begin{array}{c}\text { Pelani- } \\
\text { [us...]alif. }\end{array}$ & $\begin{array}{l}\text { h.s.e. } \\
\text { s.t.t.l. }\end{array}$ & 142 & $\begin{array}{c}\text { Monte- } \\
\text { hermoso } \\
\text { (CA) } \\
\text { [us...]ali f. }\end{array}$ \\
\hline & & & & $B a[\ldots u] x($ or $)$ & [h.s.] est & 152 & $\begin{array}{c}\text { Villar del } \\
\text { Pedroso } \\
\text { (CA) }\end{array}$ \\
\hline & Tuginocius? & & $X X X V$ & & & & $\begin{array}{l}\text { Larrodrigo } \\
\text { (SA) }\end{array}$ \\
\hline & & & & & & 177 & $\begin{array}{c}\text { Masueco } \\
\text { (SA) }\end{array}$ \\
\hline & C.D. & & & & & 198 & $\begin{array}{c}\text { Castillo de } \\
\text { Bayuela } \\
\text { (TO) }\end{array}$ \\
\hline & & & & & & 199 & $\begin{array}{l}\text { Castillo de } \\
\text { Bayuela }\end{array}$ \\
\hline & Cacus & Turi [f.] & & $\begin{array}{l}\text { Tancinus } \\
\text { lib(ertus) }\end{array}$ & p.c. & 209 & $\begin{array}{c}\text { Torralba de } \\
\text { Oropesa } \\
\text { (TO) }\end{array}$ \\
\hline
\end{tabular}


Il ne semble faire aucun doute que ces sculptures zoomorphes sont liées au monde funéraire des Vettons. Les textes, en dépit de certains qui sont illisibles, sont révélateurs de ce lien: ce sont bien des épitaphes qui sont gravés sur un ou même deux des flancs des sculptures zoomorphes, comme les n. ${ }^{\circ} 12$ et 87 . Le verraco de Larrodrigo est gravé sur le flanc gauche mais la première ligne (Tuginocio?) débute pratiquement sur le dos de l'animal; la seconde ligne (an XXXV) commence à côté de l'oreille (Martín VALLS et Frades Morera 1981 185-197; LóPEZ Monteagudo 1989 135). La consécration aux Mânes, les formules funéraires h.s.e.s.t.t.l. et f.c., l'usage du datif appliqué au premier nom qui apparaît, l'indication de l'âge, absente des autels votifs ou de tout autre document épigraphique, sont des éléments sans équivoque possible. Il n'y a aucune raison de penser que les verracos gravés d'une inscription devenue illisible, ne soient pas assimilables aux autres. De plus, les quelques inscriptions lisibles sur verracos retrouvés dans les environs immédiats de la Vettonnie (Coca, Trás-os-Montes, province de Zamora) ont toutes des inscriptions funéraires (LÓPEZ MONTEAGUDO 1989 n. $\left.{ }^{\circ} 187,227,259,266,274,277\right)$. Exception faite du support, ces textes funéraires présentent-ils une certaine originalité, un trait caractéristique? Malgré leur mauvais état de conservation, il est difficile de répondre par l'affirmative. On trouve en effet mention des éléments habituels entrant dans la composition des épitaphes, à l'exception du n. ${ }^{\circ} 56$ qui, selon G. López Monteagudo, ne se terminerait pas par f.c., ce qui est possible, mais par m(emoriae) $c(a u s a)$, interprétation qui se détache cependant de la proposition de R. C. Knapp (f.c.) (LóPEz MoNTEAGUdo 1989129 n. ${ }^{\circ}$ 56; KNAPP 199282 n. ${ }^{\circ}$ 94; RodríGUEZ AlMEIDA 1980155 n. ${ }^{\circ}$ 73: M.C.). Selon d'autres auteurs, M.C. serait l'abréviation de m(onumentum) (faciendum) c(uravit) (MARTín VAlls et PÉREZ HeRRERO 1976 70-71). Même si la trancription M.C. est exacte, cela ne remet nullement en cause la nature funéraire du document. De même, Viviros, sur l'inscription n. ${ }^{\circ} 107$, malgré son incertitude totale, peut être le nom d'un défunt ou d'un dédicant. De la même façon, sur le document n. ${ }^{\circ}$ 126, le nom que G. López Monteagudo a transcrit Ateroecon, peut se rapporter à une structure suprafamiliale (au génitif pluriel) (Azevedo 1982 321ss; Donas BotTo 1986 n. ${ }^{\circ} 72$ lit Ambroecon). Cette entité a pu être le dédicant collectif d'un "verraco-stèle" mais cette hypothèse est peu probable car Ateroecon semble être l'unique nom gravé. La statue zoomorphe peut également marquer un emplacement sacré appartenant au groupe social en question. 
Ces verracos ont été érigés dans un milieu indigène fortement prononcé. En cela, ils s'intègrent aisément dans le contexte avilais. Ce n'est pas un hasard si la plupart des verracos funéraires ont été retrouvés dans la province d'Ávila qui a fourni l'essentiel d'une statuaire zoomorphe dont il ne faut pas rechercher les premiers modèles hors de Vettonnie (ÁLVAREZ-SANCHís 1995 343-347). Le caractère indigène apparaît également dans l'onomastique. Ce sont bien des indigènes dont les noms sont gravés sur les verracos (Balarus, Cadanus, Magilo, Caurus...). A. Blanco Freijeiro évoque des "indigènes plus ou moins romanisés" (Blanco FreIJEIRo 1994 8-10). On trouve également Varus et Gaia (?) qui sont des noms latins. A la place du premier de ces noms, E. Rodríguez Almeida a proposé P(ublio) M(emmio) Vari(ano), ce qui ferait de l'individu en question un citoyen romain (RoDRíGuez AlmEIDA 1980 n. ${ }^{\circ}$ 59; ARIAS et alii 198665 n. ${ }^{\circ}$ 39). CIL II 3053 mentionne également un T. Sempronius Reburr[us] (?) mais ce verraco est aujourd'hui introuvable (KNAPP 199276 n. ${ }^{\circ}$ 87). La présence de tria nomina sur un verraco ne nous semble pas impossible, mais seulement peu probable. Et plutôt que Gaia, R. C. Knapp préfère lire Cael.t $(i) c u[m]$, ce qui prouve que l'aspect de l'inscription peut mener à des transcriptions bien différentes selon les épigraphistes (KNAPP 199277 n. ${ }^{\circ} 88$ ). S'il s'agit bien de Caelticum et nom de Gaia, nous serions en présence d'une structure suprafamiliale, peut-être une déformation ou une mauvaise lecture de Calaetiq(um), attestée sur le "verracos-stèle" de Longinus Prisco (Figure IX) lui aussi provenant de Guisando et sur une autre stèle d'Ávila (GonZÁLEZ 1986153 n. 74 et 75). Il apparaît donc qu'il faille établir un lien entre le site de Guisando et la structure suprafamiliale Calaetiqum ou Caelticum. Il est difficile d'en tirer des conclusions plus précises, mais l'hypothèse d'un espace sacré ou d'une nécropole appartenant à ce groupe social par ailleurs présent à Ávila, est tout à fait envisageable. D'autres noms de structures suprafamiliales sont gravés sur des verracos vettons: outre Ateroecon (très incertain), Caburi(qum) ou Cabura[teic](um) (ce dernier étant attesté sur une autre inscription avilaise) est lisible sur un verraco intégrée à la muraille d'Ávila (n. $\left.{ }^{\circ} 11\right)$ (GONZÁlEZ 1986152 n. 62 et 63; RodRíGUEZ AlmEIDA 1980 143-145 n. 58 propose Cabura[teic](um?) sur l'inscription du verraco soit la même structure suprafamiliale que dans l'inscription incorporée à la muraille d'Ávila); enfin [P]in[...], sans doute Pintolanqum (par ailleurs attesté dans le sanctuaire vetton de Postoloboso), pourrait être une autre de ces structures, celle à laquelle appartiendrait Titillus (RoDRíGUEZ 
AlmEIDA 1980155 n. ${ }^{\circ}$ 73; ARIAS et alii 198693 n. ${ }^{\circ}$ 67; KNAPP 1992 82 n. ${ }^{\circ}$ 94).

Enfin, sur le plan chronologique, l'examen des caractères épigraphiques fournit des dates comprises entre les Ier et IIè siècles ap. J.C.:

\begin{tabular}{|c|c|}
\hline n. ${ }^{\circ} 10$ & A partir de 100.ap. J.C. \\
\hline n. ${ }^{\circ} 11$ & Iiè s. ap.J.C. \\
\hline n. ${ }^{\circ} 12$ & Iiè s. ap.J.C. \\
\hline n. ${ }^{\circ} 27$ & Ier s. ap.J.C. \\
\hline n. ${ }^{\circ} 56$ & Fin IIè siècle ap.J.C. \\
\hline n. ${ }^{\circ} 88$ & Ier s. ap.J.C. \\
\hline n. ${ }^{\circ} 98$ & Ier s. ap.J.C. \\
\hline n. ${ }^{\circ} 173$ & IIè s. ap.J.C. \\
\hline
\end{tabular}

Si ces verracos sont effectivement des stèles funéraires, bien qu'aucune urne cinéraire contemporaine n'ait été retrouvée à proximité immédiate (mais c'est également le cas pour les stèles funéraires), n'étaient-il pas autre chose que de simples supports pour les épitaphes?

La théorie selon laquelle ces verracos faisaient partie d'un monument cinéraire est intéressante et est corroborée par des documents archéologiques retrouvés dans divers sites de la province d'Ávila, en particulier à Martiherrero (MARTín VALLS et PÉREZ HERRERo 1976 67-88; Martín Valls et Pérez Herrero 1994 403-416).

A Ávila, la muraille a révélé des traces d'éléments architecturaux bien particuliers: il s'agit vraisemblablement de cistae sur lesquels devaient reposer des cupae portant des épitaphes ou bien des statues zoomorphes dont certaines sont reconnaissables, incorporées à la muraille, mais dont il est difficile de dire si elles portaient des inscriptions funéraires. Il semblerait que l'église San Miguel de Arévalo et le site avilais de Riofrío aient également livré des verracos et des blocs granitiques ressemblant à des cistae (MARTín VALLs 1974 69-82; LÓPEZ MonTEagudo 1989 11). 
A Martiherrero, site proche d'Avila, quatre verracos ont été localisés à proximité de blocs de granite, vraisemblablement des cistae, équipés d'un petit canal aboutissant à une cavité creusée dans le bloc, sans doute à des fins de libations (Martín Valls et Pérez HerRero 1976 67-80). Une des cavités a révélé la présence des restes de la crémation du défunt et de ses objets personnels, peut-être à l'époque de Clodius Albinus (196-197 ap. J.C.), d'après une monnaie retrouvée parmi le mobilier funéraire. Parmi les cendres, ont été identifiés des os calcinés, un morceau de crâne humain, des fragments de verre, de bronze, un morceau de lame de couteau en fer, des clous, des tessons de céramique (Martín Valls et Pérez Herrero 1976 74-75).

Il nous faut donc admettre que ces verracos gravés faisaient partie de monuments funéraires renfermant les cendres de défunts. Des questions restent cependant sans réponse: les autres verracos vettons, anépigraphes, avaient-ils le même usage? S'agit-il de réutilisations de verracos protohistoriques qu'on a gravé à l'époque romaine ou bien peut-on imaginer que l'ensemble du monument funéraire ait été confectionné sous le Haut-Empire romain? Mais surtout, comment interpréter un tel usage: simple pratique funéraire en usage dans certains milieux (par exemple celui des propriétaires de bétail ou encore celui des prêtres chargés des sacrifices des animaux) ou bien réelle motivation religieuse liée à une signification symbolique du porc? Enfin, l'usage funéraire de ces verracos explique-t-il qu'aucune stèle vettonne ne soit décorée d'une figure de porc ou de taureau?

Ainsi, au terme de cette étude sur les traces épigraphiques et archéologiques du monde funéraire vetton, il nous faut conclure qu'au sein de la culture matérielle des Vettons, ces témoignages demeurent difficiles d'interprétation pour les raisons que nous avons évoquées, la plus importante étant l'absence de contexte archéologique des stèles funéraires (d'où la difficile mise en relation entre le rite de l'incinération et l'usage des stèles funéraires) et la très difficile interprétation des motifs présents sur ces dernières. Les lacunes des sources littéraires ne nous permettent pas de combler ce vide. En l'état actuel des connaissances, il ne nous est même pas possible de fournir par exemple une chronologie précise des rites d'incinération et d'inhumation en pays vetton ou de mettre cette dernière pratique avec le développement du christianisme. On peut seulement envisager la probabilité d'une forte résistance de l'incinération, sa permanence dans certaines régions et/ou milieux sociaux et affirmer sans trop de doutes la cohabitation des deux 
rites dans une même nécropole. L'étude détaillée des stèles nous permet de mettre en relief plusieurs grands groupes de types de stèles (groupe salmantin, groupe avilais...) qui, outre les cas atypiques de stèles d'indigènes copiant plus ou moins maladroitement des modèles romains, font apparaître une Vettonnie à la fois manquant d'unité culturelle et très perméable aux influences voisines (astures, lusitaniennes, carpétanes), phénomène que nous avions déjà mis en relief avec l'étude des divinités indigènes. En ce sens, il ne semble pas exister d'art ni de pratiques funéraires en relation directe avec la culture vettonne, à l'exception de l'usage des verracos à des fins funéraires, attestant là d'une réelle originalité puisque les "verracos-stèles" retrouvés hors de Vettonnie se situent tous en bordure du pays vetton.

\section{BIBLIOGRAPHIE}

Abascal Palazón, José Manuel (1994) - Los nombres personales en las inscripciones latinas de Hispania, Murcia.

ABÁsolo, José Antonio et MARCo, Francisco (1995) - "Tipología e iconografía en las estelas de la mitad septentrional de la península ibérica" Roma y el nacimiento de la cultura epigráfica en Occidente (Zaragoza, 1992), Zaragoza.

Abásolo, José Antonio, Albertos, María Lourdes et EloRZA, J.C. (1975) - Los monumentos funerarios de la época romana en forma de casa de la región de Poza de la Sal (Bureba, Burgos), Burgos.

ACuÑa CASTRoviejo, F. (1974) - "Notas sobre la morfología y la decoración en las aras y estelas de Galicia en época romana" Studia Archaeologica 32 17-31.

Aguilar, Antonio et Guichard, Pascal (1993) - Villas romaines d'Estrémadure. Doña María, La Sevillana et leur environnement, Madrid.

Aguilar, Antonio et Guichard, Pascal (abril de 1993) - "Lacimurga. La ciudad antigua y su entorno" Revista de Arqueología 144 32-38.

ALARCÃo, Jorge de (2001) - "Novas perspectivas sobre os Lusitanos (e outros mundos)" Revista Portuguesa de Arqueologia 42 293-349.

Alonso, A. et CRESPO, Santos (1999) - Corpus de inscripciones romanas de la provincia de Salamanca. Fuentes epigráficas para la historia social de Hispania romana, Valladolid.

Álvarez, A. et García-Hoz, M.C. (1988) - Museo de Cáceres. Guía breve de la sección de Arqueología, Cáceres.

ÁlvareZ-SANChís, J.R. (1995) - "Esculturas de verracos y etnicidad en el contexto de la romanización” XXII CNA II (Vigo, 1993), Vigo.

ÁlvareZ-Sanchís, J.R. (1999) - Los Vettones (Bibliotheca Archaeologica Hispana 1), Madrid. 
ARIAS, P., LóPEZ, M. et SÁNCHEZ J. (1986) - Catálogo de la escultura zoomorfa protohistórica y romana de tradición indígena de la provincia de Ávila, Ávila.

Audin, A. (1960) - "Inhumations et incinération" Latomus 19 312-322.

Azevedo, R. (1982) - “O porco na zoolatria ibérica” TAE 242 321-329.

BALIL, A. (1970) - "La defensa de Hispania en el Bajo Imperio. Amenaza exterior e inquietud interna" Legio VII Gemina, León, 603-620.

BALIL, A. (1992) - "Aspectos de la vida y la muerte en la antigua Hispania" Religio Deorum. Actas del Coloquio Internacional de Epigrafía: culto y sociedad en Occidente (Tarragona, 1988), Sabadell.

Bel, V., Tranoy, L., Beraud, I. et Gebara, C. (1992) - "Les nécropoles à incinération et à inhumation en Gaule méridionale" Incinérations et inhumations dans l'Occident romain aux trois premiers siècles de notre ère (France, Espagne, Italie, Afrique du Nord, Suisse, Pays-Bas, Grande-Bretagne). Actes du IVè Congrès archéologique de Gaule méridionale (Toulouse, 1987), Toulouse, 9-29.

BELTRÁn Lloris, Miguel (1975-76) - "Aportaciones a la epigrafía y arqueología romana de Cáceres” Caesaraugusta 39-40 19-111.

Bendala Galán, J., (1992) - "Incinérations et inhumations dans l'Occident romain aux trois premiers siècles de notre ère: le sud de l'Espagne" Incinérations et inhumations dans l'Occident romain aux trois premiers siècles de notre ère (France, Espagne, Italie, Afrique du Nord, Suisse, Pays-Bas, Grande-Bretagne). Actes du IVè Congrès archéologique de Gaule méridionale (Toulouse, 1987), Toulouse, 77-90.

Blanco Freijeiro, A. (1994) - "Museo de los verracos celtibéricos" BRAH 181 1-60. BlázQuez Martínez, José María (1965) - Cáparra I (EAE 34), Madrid.

Blázquez Martínez, José María (1965a) - "La Tomba del Cardinale y la influencia orfico-pitagórica en las creencias estruscas del Ultratumba” Latomus 24 3-39.

BlázQuez MARTíneZ, José María (1975) - Diccionario de las religiones prerromanas de Hispania, Madrid.

Blázquez Martínez, José María (1983) - Primitivas religiones ibéricas II: Religiones prerromanas, Madrid.

Blázquez Martínez, José María (1985) - "Magia y religión entre los pueblos indígenas de la Hispania antigua” Religión, superstición y magia en el mundo romano, Cádiz.

Bonnaud, Christophe (1999) - Vettonia Antiqua. Peuplement et société dans l'ouest de la Meseta, thèse dactylographiée, 5 vol., Poitiers.

Bonnaud, Christophe (2002) - "Vettonia Antiqua: les limites ethniques et administratives d'un peuple de l'ouest de la Meseta dans l'Antiquité" SHHA 20 171-199.

Bonnaud, Christophe (2002a) - "Les divinités indigènes de Vettonnie sous le Haut-Empire romain: essai d'inventaire et interprétation" Conimbriga 41 63-103. Botтo, Donas, M. M. (1986) - "Inscrição rupestre de Penela da Beira" FE 1672.

BRELICH, A. (1937) - Aspetti della morte nelle iscrizioni sepolcrali dell'imperio romano, Budapest.

Bost, Jean Pierre et Maurin, Louis (1992) - "Incinération et inhumation entre la Loire et les Pyrénées” Incinérations et inhumations dans l'Occident romain aux trois 
premiers siècles de notre ère (France, Espagne, Italie, Afrique du Nord, Suisse, Pays-Bas, Grande-Bretagne). Actes du IVè Congrès archéologique de Gaule méridionale (Toulouse, 1987), Toulouse, 41-65.

CABRÉ, M.E. (1953) - "El simbolismo solar en la ornamentación de las espadas de la II Edad del Hierro Céltico de la Península Ibérica" APL 3 101ss.

Cabré Aguiló, J., Cabré de Morán, E. et Molinero Pérez A. (1950) - El castro y la necrópolis del Hierro céltico de Chamartín de la Sierra (Ávila) (Acta Arqueológica Hispánica V), Madrid.

CAllejo Serrano, C. (1963) - "Fichas de arqueología extremeña" AEA 36 107/108 222-228.

Callejo Serrano, C. (1967) - "Cédulas epigráficas del campo norbense" Zephyrus 18 85-119.

Callejo Serrano, C. (1969-1970) - "Excavaciones realizadas en la Cerca de los Hidalgos, Campolugar (Cáceres) NAH 13-14 36-51.

Callejo Serrano, C. (1977) - "Simbología funeraria romana de la Alta Extremadura" Revista de la Universidad Complutense de Madrid (Homenaje a García y Bellido III) 26 145-161.

Canto, Alicia María, Bejarano, A. et Palma, F. (1997) - "El Mausoleo del Dintel de los Ríos de Mérida, Revve Anabaraecus y el culto de la confluencia" MDAI (M) 38 247-296.

Castelo, R. et SÁnchez, E. (1995) - "De verribus Vettonum. El verraco de Talavera de la Nueva (Toledo) y algunas notas sobre la arqueología de las tierras orientales vetonas" Zephyrus 48 317-330.

Cedillo, C. de (1959) - Catálogo Monumental de la Provincia de Toledo, Toledo.

Cerdeño, M.L. et CABANES, E. (1994) - "El simbolismo del jabalí en el ámbito céltico peninsular" TP 512 103-109.

CERRILLo, Enrique (1980) - "Notas sobre cerámica funeraria (siglos IV-VIII). La colleción de jarros del Museo Arqueológico de Cáceres" Miscelánea Cacereña (primera serie), Cáceres, 51-64.

CORBIER, Paul (1998) - L'épigraphie latine, Paris.

CorTés, S., FERnÁNDEZ F.J. et OCAÑA E. (1989-90) - "Cuatro inscripciones empotradas en las murallas de Talavera de la Reina (Toledo)" Norba 10 67-77.

Coulon, Gérard (juin 1987) - "La cérémonie des funérailles en Gaule romaine" L'Histoire $10126-32$.

CREspo Ortiz de ZÁrate, Santos et Alonso, Á. (1999) - Las manifestaciones religiosas del mundo antiguo en Hispania romana: el territorio de Castilla y León I: Las fuentes epigráficas, Valladolid.

Crespo Ortiz de ZÁrate, Santos et Alonso, Á. (1999a) - "La precisión en la edad de la muerte en la epigrafía funeraria de Hispania" J. ALvar (ed.) Homenaje a José María Blázquez vol. IV: Hispania romana I, Madrid, 95-119.

Cumont, F. (1942) - Recherches sur le symbolisme funéraire des Romains, Paris. Curado, F. (1988) - "Estelas funerarias de Vila Boa (Sabugal)" FE 271231.

CURCHIN, Leonard A. (1997) - "Funerary customs in Central Spain: the transition from pre-Roman to Roman practice" Hant 21 7-34. 
Curchin, Leonard A. (2000) - "La famille lusitano-romaine" Sociedad y cultura en Lusitania romana (IV Mesa Redonda Internacional, Mérida, 2000) 329-341. EDMONDSON, J. (2000) - "Conmemoración funeraria y relaciones familiares en Augusta Emerita" Sociedad y cultura en Lusitania romana (IV Mesa Redonda Internacional, Mérida, 2000), Mérida, 299-327.

Edmondson, J., Nogales, T. et Trillmich, W., Imagen y memoria: monumentos funerarios con retratos de Augusta Emerita (Monografías Emeritenses) (sous presse). ETIEnNe, R. (1973) - "Les syncrétismes religieux dans la Péninsule Ibérique à l'époque impériale" Colloque sur les syncrétismes dans les religions grecque et romaine (Strasbourg, 1971), Paris, 153-163.

FERnÁndez Gómez, F. (1997) - La Necrópolis de la Edad del Hierro de "El Raso" (Candeleda, Ávila). Las Guijas B (Junta de Castilla y León, Memorias), Zamora. Floriano Cumbreño, A.C. (1944) - "Excavaciones en la antigua Caparra, Cáparra, Cáceres" AEA 18 270-286.

Fuentes Domínguez, Á. (1989) - La necrópolis tardorromana de Albalete de las Nogueras (Cuenca) y el problema de las denominadas "necrópolis" del Duero, Cuenca.

Fuentes Domínguez, Á. (1992) - "Incinérations et inhumations dans la moitié nord de l'Espagne au cours des trois premiers siècles de notre ère" Incinérations et inhumations dans l'Occident romain aux trois premiers siècles de notre ère (France, Espagne, Italie, Afrique du Nord, Suisse, Pays-Bas, Grande-Bretagne). Actes du IVè Congrès archéologique de Gaule méridionale (Toulouse, 1987), Toulouse, 91-106.

Gamallo, J.L. et Gimeno, H. (1990) - "Inscripciones del norte y sudoeste de la provincia de Cáceres: revisión y nuevas aportaciones" CuPAUM 18 277-306. GARCíA de Figuerola, M. (1984-1985) - "Una tumba tardo-romana con ajuar en Valverde del Fresno (Cáceres)" Zephyrus 37-38 371-375.

García Gelabert, M.P. et BlÁzQuez, J.M. (1994) - "Estelas funerarias con retratos" C. DE LA CASA (ed.) Actas del V Congreso Internacional de Estelas Funerarias (Soria,1993), Soria, 309-321.

GARCía y BELLIDO, Antonio (1967) - "Sobre un tipo de estela funeraria de togado bajo hornacina" AEA 40 115/116 110-120.

Gómez Pantoja, J. (1999) - "Historia de dos ciudades: Capera y Clunia" J.G. Gorges et F.G. RodríGUEZ (éd.) Economie et territoire en Lusitanie romaine, Madrid, 91-108 .

GonzÁlez Cordero, A. (1989) - "Las tumbas excavadas en la roca en la provincia de Cáceres" Alcántara 171989 137ss.

GonzÁlez RodríGUEz, M. C. (1986) - Las unidades organizativas indígenas del área indoeuropea de Hispania (Anejos de Veleia 2), Vitoria, 1986.

Gordon, A.E. (1983) - Illustrated Introduction to Latin Epigraphy, Berkeley - Los Angeles - Londres.

Graf, F. (1994) - La magie dans l'Antiquité romaine. Idéologie et pratique, Paris.

HABA QuiRós, S. et Rodrigo LóPEZ, V. (1986) - “Aportaciones a la epigrafía latina del área caparense" Norba 7 43-60. 
HATT, Jean-Jacques (1981) - "Images indigènes et images gréco-romaines dans l'expression des conceptions religieuses celtiques" G. SiEBERT (éd.) Méthodologie iconographique. Actes du colloque de Strasbourg (1979), Strasbourg, 59$-62$.

HAtT, Jean-Jacques (1986) - La tombe gallo-romaine, Paris.

Hernández, L., MaÑanes, T. et Jiménez de Furundarena, A. (1994) - "Nuevas aportaciones a la epigrafía salmantina: Hinojosa de Duero" Hant 18 317-379.

HERnANDo Sobrino, M. R. del (1994) - Indigenismo y romanización en la provincia de Ávila, thèse dactylographiée, Madrid.

Hernando Sobrino, M. R. del (1999) - "Epigrafía abulense: novedades" Homeneje al profesor Montenegro (Estudios de Historia antigua), Valladolid, 531-537.

Hopkins, K. (1987) - “Graveyards for historians” F. HinARD (dir.) La mort, les morts et l'au-delà dans le monde romain. Actes du Colloque de Caen (1985), Caen, 113-126.

Hurtado de SAn Antonio, R. (1979) - Corpus provincial de las inscripciones latinas (Cáceres) Cáceres.

JimÉnEZ DE FurundANERA, A. (1993) - “Contribución a la epigrafía del Oeste de la provincia de Salamanca. El conjunto de Médina del Campo (Valladolid)" ETF (Serie 2) 6 133-160.

KNAPP, R.C. (1984) - "The epigraphic evidence for native and roman religion in Iberia" Acta of the 8th International Epigraphic Congress (Athens, 1981), Athènes, 219-230 .

KNAPP, R.C. (1992) - Latin Inscriptions from Central Spain (Classical Studies, 34), Berkeley - Los Angeles - Oxford.

Kooy, C. (1981) - "Le croissant lunaire sur les monuments funéraires gallo-romains" Gallia 39 45-62.

LAFON, Xavier (2002) - "La localisation des monuments funéraires" La mort des notables en Gaule romaine (Catalogue de l'exposition), Lattes, 113-120.

LAMBrino, Scarlat (1965) - "Les cultes indigènes en Espagne sous Trajan et Hadrien" Les empereurs romains d'Espagne (Madrid, 1964), Paris, 223-242.

LASSÈRE, Jean-Marie (1987) - "Difficultés de l'estimation de la longévité. Questions de méthode" F. HinARD (dir.) La mort, les morts et l'au-delà dans le monde romain. Actes du Colloque de Caen (1985), Caen, 91-97.

LE RouX, Patrick (1982) - L'armée romaine et l'organisation des provinces ibériques d'Auguste à l'invasion de 403, Paris.

López Monteagudo, Guadalupe (1982) - "Las esculturas zoomorfas "célticas" de la península ibérica y sus paralelos polacos" AEA 55 3-30.

LóPez Monteagudo, Guadalupe (1989) - Esculturas zoomorfas celtas de la península ibérica (Anejos de AEA, 10), Madrid.

Lozano Velilla, A. (1973) - "Tipología de las estelas y población de Hispania" Revista de la Universidad Complutense de Madrid (Separata) 2286 89-114. Maluquer de Motes, J. (1951) - "De la Salamanca primitiva" Zephyrus 2 61-72. Maluquer de Motes, J. (1956) - Carta arqueológica de España. Salamanca, Salamanca. 
MALuQuer de Motes, J. (1968) - "Excavaciones arqueológicas en el Cerro de Las Merchanas (Lumbrales, Salamanca)" Pyrenae 4 101-128.

Mangas Manjarrés, J. (1978) - "Religiones indígenas en Hispania" J.M. BlázQuez et alii Historia de España Antigua 2: Hispania romana, Madrid, 625-661.

Mangas, J. et Carrobles, J. (1992) - "La ciudad de Talavera de la Reina en época romana" Actas de las Primeras Jornadas de Arqueología de Talavera de la Reina y sus tierras (Talavera de la Reina, 1990), Toledo, 95-138.

Marco Simón, Francisco (1976) - Tipología y técnica en las estelas decoradas de los conventos Caesaraugustano y Cluniense, Zaragoza.

Marco Simón, Francisco (1978) - Las estelas decoradas de los conventos caesaraugustano y cluniense, Zaragoza.

Marco Simón, Francisco (1985) - "Mentalidad funeraria en el norte de la península ibérica en época romana" Asimilación y resistencia a la romanización en el norte de Hispania, Vitoria, 47-69.

Marco Simón, Francisco (1987) - "La religión de los Celtíberos I" Primero Symposium sobre los Celtíberos I (Daroca 1986), Zaragoza, 55-74.

Marco Simón, Francisco (1991) - "Mundo religioso y funerario" J.A. GARCÍA CASTRO (dir.) Los Celtas en la Péninsula Ibérica (RevArq hors série), Madrid, 92-101.

Marco Simón, Francisco (1993) - "La religiosidad en la Céltica hispana” M. AlmaGro-Gorbea (dir.) Los Celtas: Hispania y Europea, Madrid, 477-502.

MARTín GiL, T. (1932) - “El arte en Extremadura.Una excursión a Monroy” Revista del Centro de Estudios extremeños 6.

MARTín VALLS, Ricardo (1971) - "Inscripción romana del Bercia, Alcolea del Tajo" BSAA 37 425-429.

MARTín VALLS, Ricardo (1974) - "Variedades tipológicas en las esculturas zoomorfas de la Meseta" SA 32 69-92.

MARTín VALls, Ricardo (1976) - "Nuevos hallazgos arqueológicos en Ciudad Rodrigo" Zephyrus 26-27 374-388.

MARTín VALLs, Ricardo (1979) - "Novedades epigráficas en el castro de Yecla de Yeltes (Salamanca)" Estudios dedicados a C. Callejo Serrano, Cáceres, 1979 499-510. Martín VALls, Ricardo (1982) - "Las necrópolis del castro de Yecla de Yelte. Datos arqueológicos y epigráficos para su estudio" Zephyrus 34-35 181-201.

Martín VAlls, R. (1982a) - "Estela romana procedente de Villar de la Yegua (Salamanca)" Zephyrus 34-35 214-217.

Martín Valls, Ricardo, Benet, N. et Macarro, C. (1992) - “Arqueología de Salamanca" Primer Congreso de Historia de Salamanca I, Salamanca, 87-115.

Martín VAlls, Ricardo et Frades Morera, J. (1981) - "Un verraco con inscripción latina en Larrodrigo (Salamanca)" Numantia 1 185-197.

Martín Valls, Ricardo et Pérez Herrero, E. (1994) - “Zoomorphic Iron Age Sculpture in Western Iberia: Symbols of Social and Cultural Identity?" Proceedings of the Prehistoric Society 55 403-416.

Molano, J. et Alvarado, M. de (1991-92) - "El enterramiento de la c/I Circo Romano n. ${ }^{\circ}$ 10: aportación al conocimiento de las tumbas con tubos de libaciones en Augusta Emerita" Anas 4-5 161-173. 
Molano BríAS J. et alii, (1991-92) - "Excavaciones de urgencia en la necrópolis de la ermita de Santa Ana (Monroy)" Norba 11-12 141-170.

MorÁn BARDón, César (1922) - Epigrafía salmantina, Salamanca.

MorÁn SÁNCHEZ, C.J. s.d. - Augustobriga: análisis arqueológico de una peculiar ciudad romana en Hispania, Mémoire dactylographié, Cáceres.

Morris, I. (1992) - Death Ritual and Social Structure in Classical Antiquity, Cambridge. NAVArro Caballero, Milagros (2000) - "Notas sobre algunos gentilicios romanos de Lusitania: una propuesta metodológica acerca de la emigración itálica” Sociedad y cultura en Lusitania romana (IV Mesa Redonda Internacional, Mérida, 2000), Mérida, 281-297.

NAVAscuÉs, J.M. de (1963) - "Caracteres externos de las antiguas inscripciones salmantinas. Los epitafios de la zona occidental” BRAH 152 159-223.

Nogales Basarrate, T. (1994) - "Las estelas funerarias peninsulares en el mundo clásico. El fenómeno emeritense" C. DE LA CASA (ed.) Actas del V Congreso Internacional de Estelas Funerarias (Soria,1993), Soria, 201-210.

Nogales Basarrate, T. (1997) - El retrato privado de Augusta Emerita, Badajoz 1997.

Palol, Pedro de (1958) - "Las excavaciones de San Miguel del Arroyo. Un conjunto de necrópolis tardorromanas en el valle del Duero" BSAA 24 209-217.

PALOL, Pedro de (1964) - "Cuchillo hispanorromano del siglo IV de J.C.” BSAA 1964 67-102.

PALOL, Pedro de (1966) - "Demografía y arqueología hispánicas. Siglos IV-VIII" BSAA 32 5-66.

PIÑEL, C. (1981) - "El yacimiento tardo-romano de El Soto de Calvarrasa de Abajo (Salamanca)" Zephyrus 32-33 217-225.

PRIEUR, Jean (1986) - La mort dans l'Antiquité romaine, Rennes.

Pujol, A. (1974) - "Lápida funeraria de Monroy (Cáceres) con símbolo solar” REE 30 1 185-193.

REDONDO RodríGUez, José Antonio (1987) - Protohistoria y romanización de la Regio Turgaliensis, thèse dactylographiée, 2 vol., Cáceres.

Rodríguez AlmeIdA, Emilio (1980) - Ávila romana, Ávila.

Rodríguez Lage, S. (1974) - Las estelas funerarias de Galicia en la época romana Orense.

RodríGuez NeILA, J.F. (1976) - “Consideraciones sobre el concepto de vicus en la Hispania romana. Los vici de Corduba” Corduba 21 (2) 10-118.

SAlinAS DE FríAs, M. (1994) - "El poblamiento rural antiguo de la provincia de Salamanca:modelos e implicaciones históricas" El medio rural en Lusitania. Formas de habitat y ocupación del suelo (Mesa Redonda Internacional, Salamanca, 1993) (SHHA 10-11), Salamanca, 177-188.

SALINAS DE FríAs, Manuel (1995) - "Los inicios de la epigrafía en Lusitania oriental" Roma y el nacimiento de la cultura epigráfica en Occidente (Zaragoza, 1992), Zaragoza, 281-292.

SALINAS DE FríAs, Manuel (2001) - Los Vettones. Indigenismo y romanización en el occidente de la Meseta, Salamanca. 
SALMON, Pierre (1987) - "Les insuffisances du matériel épigraphique sur la mortalité dans l'Antiquité romaine" F. Hinard (dir.) La mort, les morts et l'au-delà dans le monde romain. Actes du Colloque de Caen (1985), Caen, 99-112.

SÁnchez Albalá, J. I. et Vinagre Nevado, D. (1998) - Corpus de inscripciones latinas de Coria (Temas Caurienses 1), Coria.

Sánchez Cabañas, A. (1967) - Historia de Ciudad Rodrigo, Salamanca.

SÁnchez Moreno, Eduardo (1997) - “Aproximación a la religión de los Vetones: Dioses, ritos y santuarios” SZ (Segunda Etapa) 4 115-147.

SÁnchez Moreno, Eduardo (2000) - Vetones: historia y arqueología de un pueblo prerromano (Ediciones de la Universidad autónoma de Madrid 64), Madrid.

Santos SÁnchez, M. (1993) - Historia de Talavera la Vieja, la romana Augustobriga, Talavera de la Reina.

Saquete Chamizo, J.C. (1977) - Las elites sociales de Augusta Emerita (Cuadernos Emeritenses 13), Mérida.

SERrano Delgado, J.M. (1987-1988) - "La aportación de la epigrafía para el conocimiento de la amicitia - relación de dependencia en el alto imperio" Habis 18-19 345-364.

Solana SÁInz, J.M. et Hernández Guerra, L. (2000) - Religión y sociedad en época romana en la Meseta septentrional, Valladolid.

SopeñA, G. (1987) - Dioses, ética y ritos. Aproximaciones para una comprensión de la religiosidad de los pueblos celtíberos, Zaragoza.

Stylow, A. U. (1995) - "Los inicios de la epigrafía latina en la Bética. El ejemplo de la epigrafía funeraria" Roma y el nacimiento de la cultura epigráfica en Occidente (Zaragoza, 1992), Zaragoza, 219-238.

TARradel, M. (1980) - "Primeras culturas. Los pueblos del centro y del norte de la península” M. TUÑON DE LARA (dir.) Historia de España 1, Barcelona.

Tranoy, Alain (1981) - La Galice romaine. Recherche sur le nord-ouest de la péninsule ibérique dans l'Antiquité, Paris.

Tranoy, Laurence (2002) - "Pratiques funéraires en Gaule romaine" Catalogue de l'exposition "La mort des notables en gaule romaine”, Lattes, 105-112.

TuRCAN, Robert (1958) - “Origines et sens de l'inhumation à l'époque impériale” REA 60 323-347.

TURCAN, Robert (1978) - "Les sarcophages et le problème du symbolisme funéraire" ANRW 216 (2) 1700-1735.

WOLSKI, W. et BerciU, I. (1973) - "Contribution au problème des tombes romaines à dispositif pour les libations funéraires” Latomus 322 371-379. 


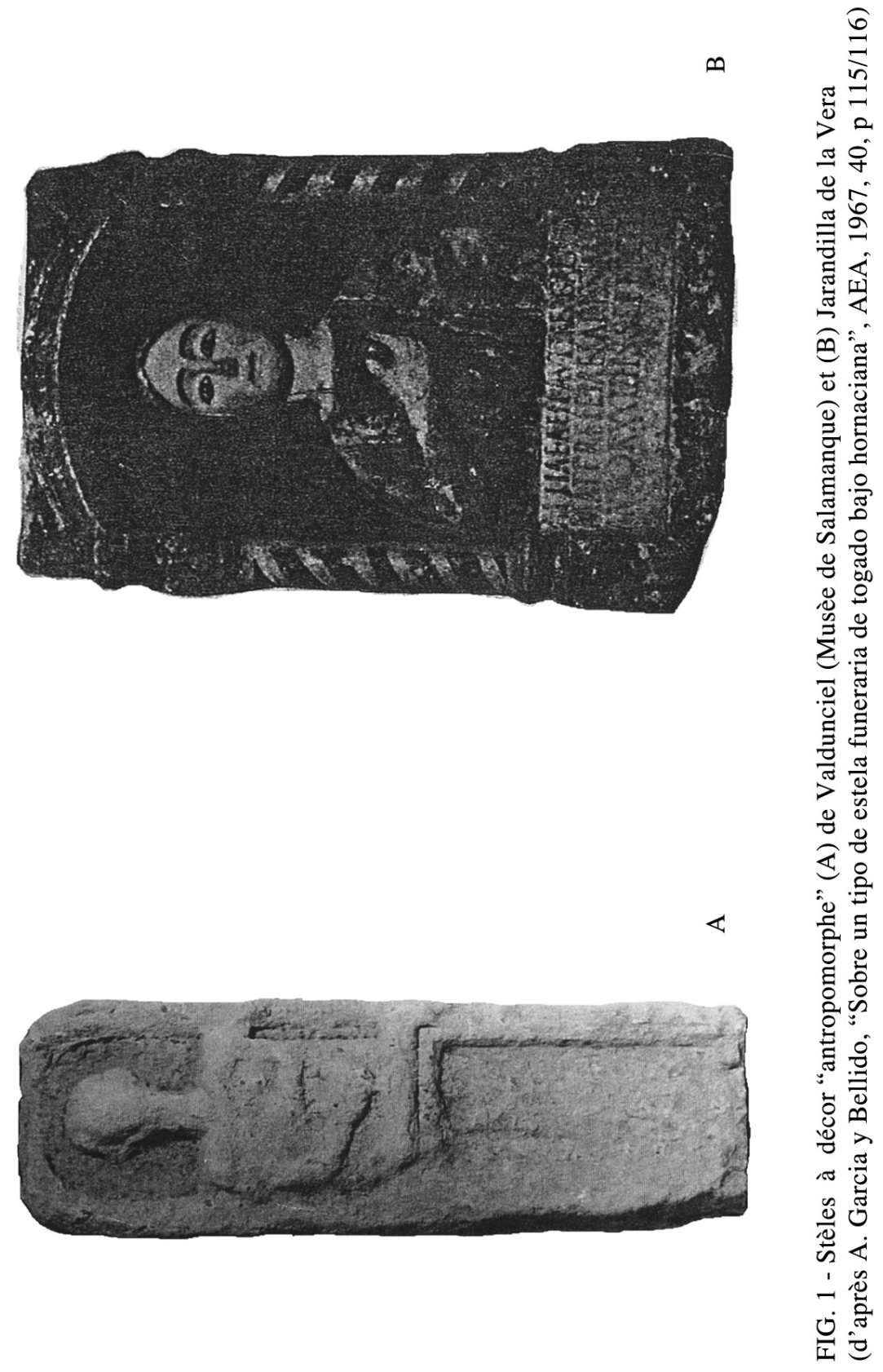



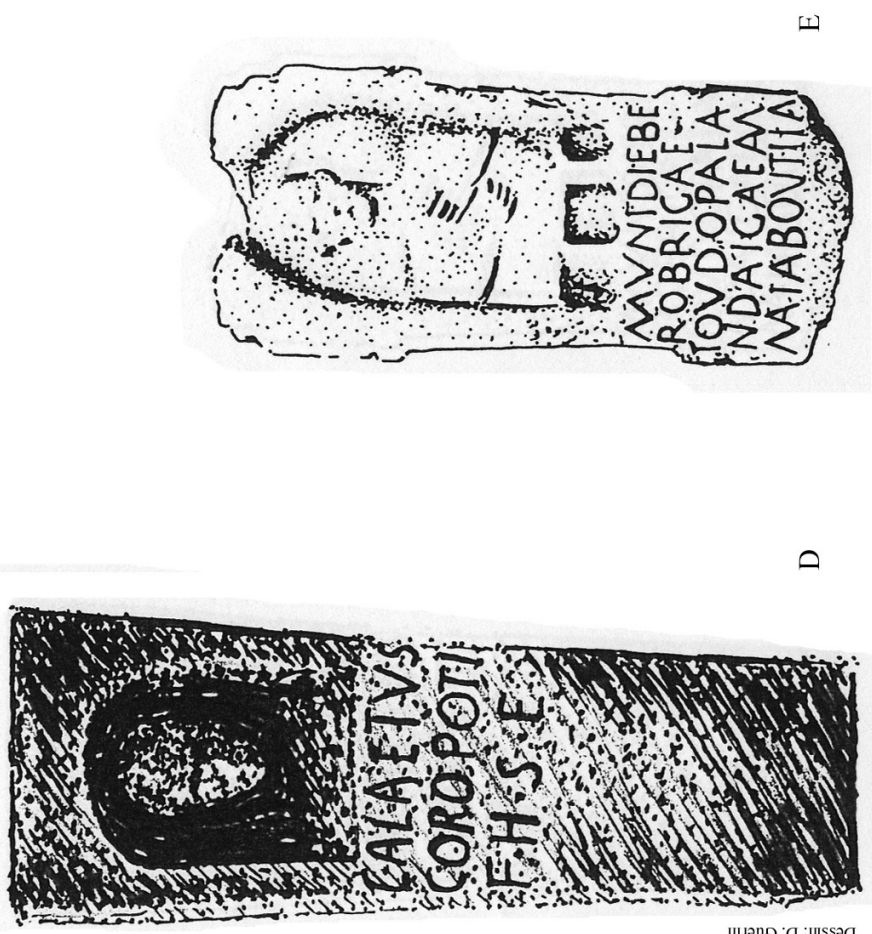

효욜

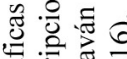

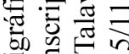
으의 ‡ 80 吾实 웡 응

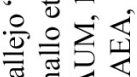
ठ है ن क ب के is 둘응 हิ चे 웡 ฮิ 훙 중 त्र 过寻 品入 站 을 ข - $0 \ddot{0}$ 는 인 $\therefore$ 究气 むU』 응 슬 ลิ ล 등 은 亟 元 ப워워 过 क क षै क ڤ 융 包 1 ( ) ่유을

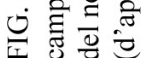




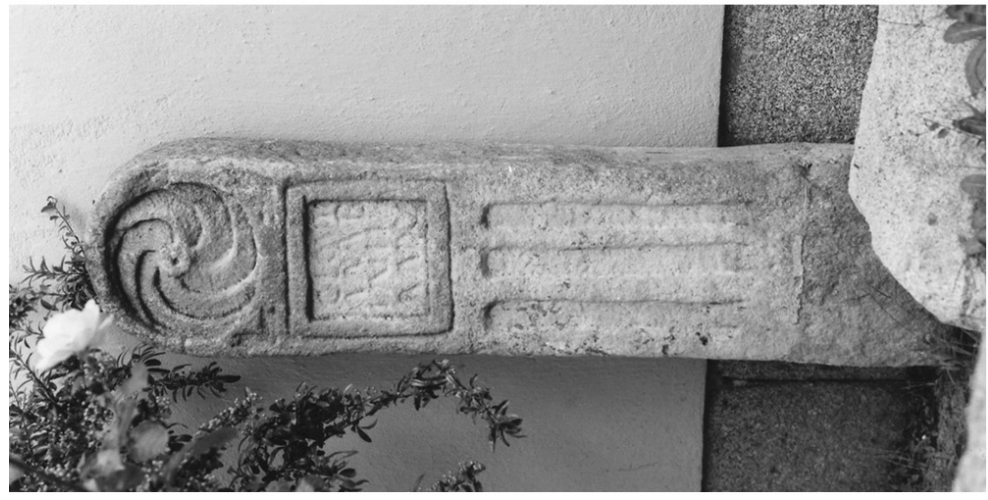

Oे

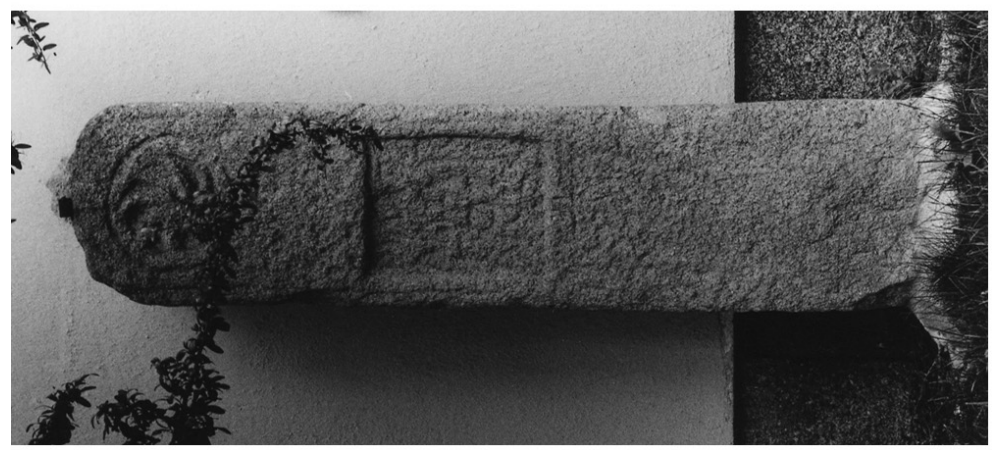




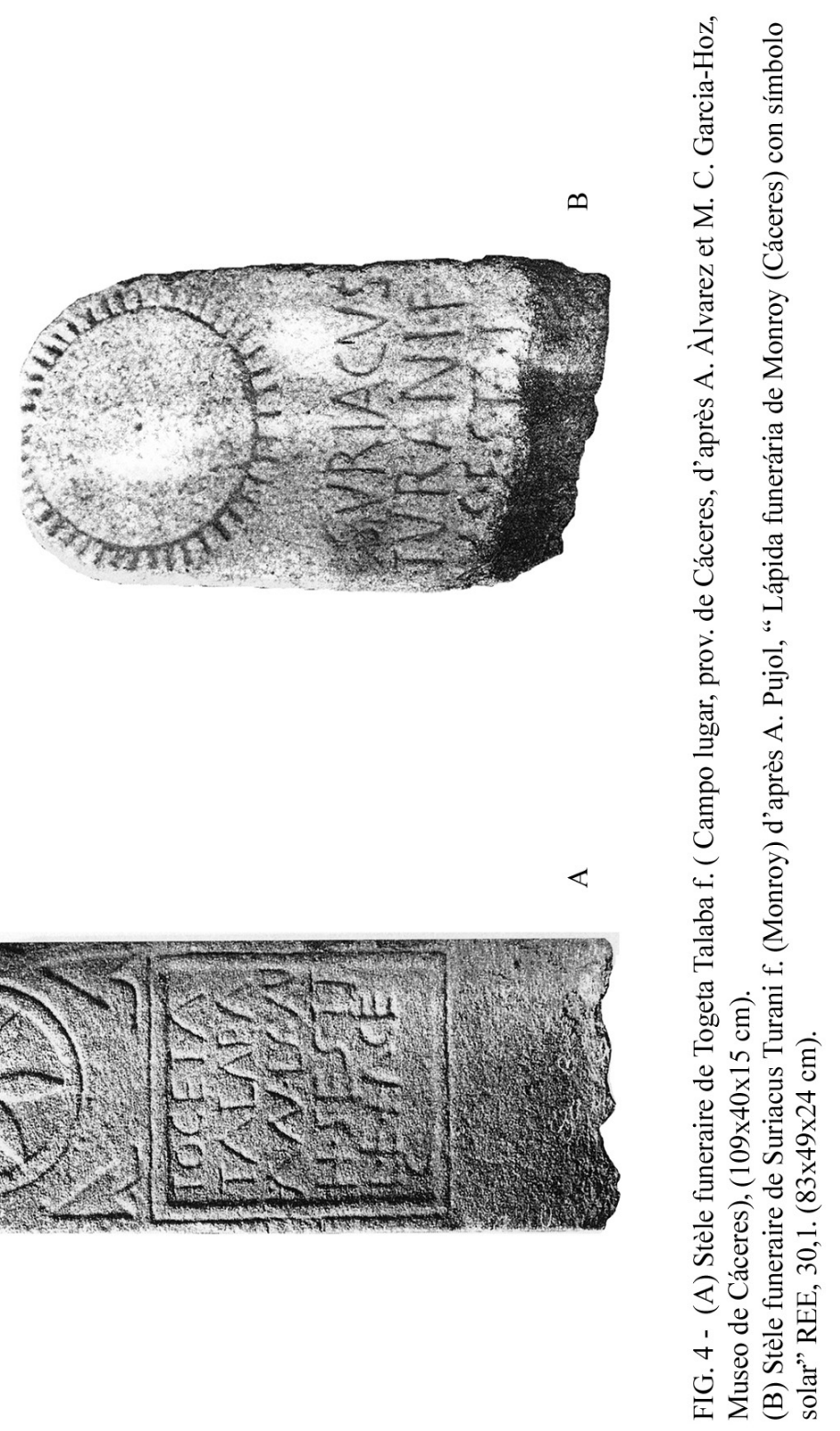




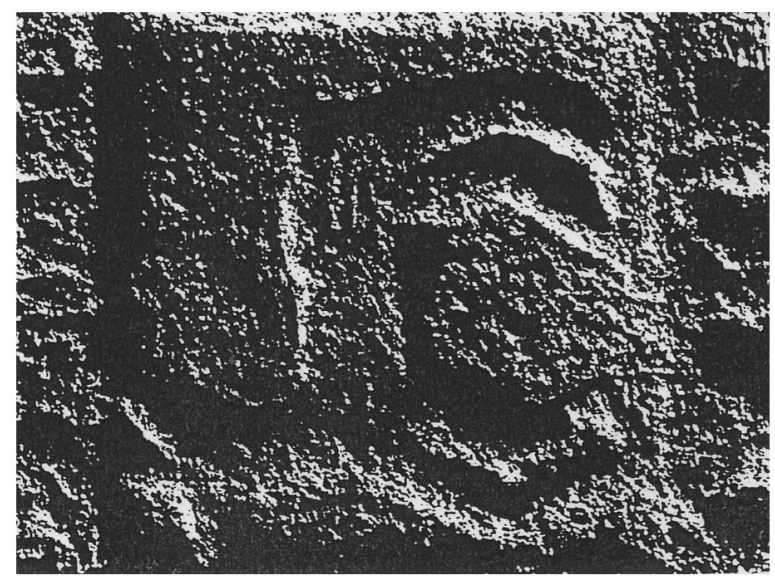

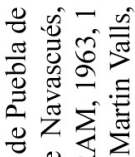

뭄

踏兽。

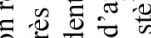

응 흥혀

है 已 व

䠌

ษ 㞼

की

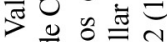

.

卷言的

ن $\dot{2} \approx 0$

क ल क व

幽宗

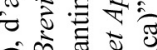
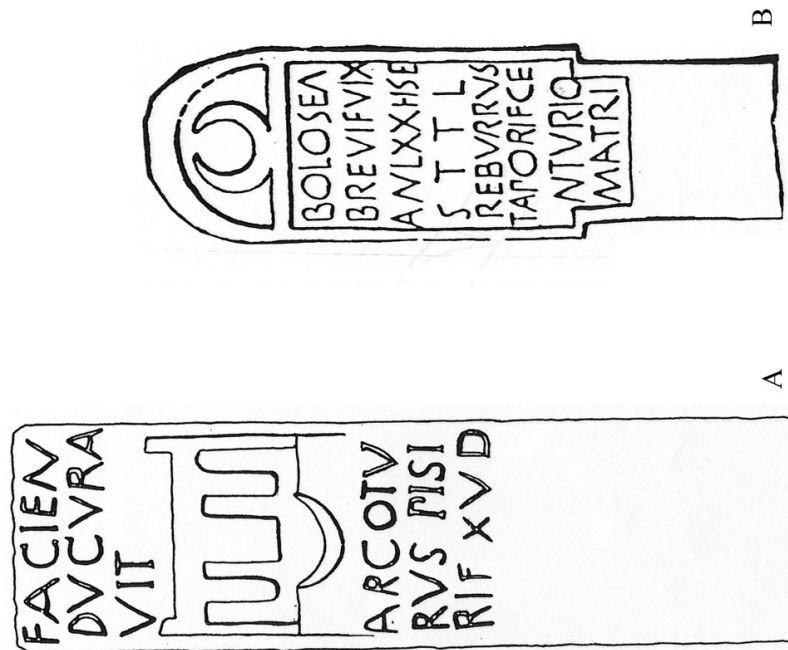

$\ll$ 

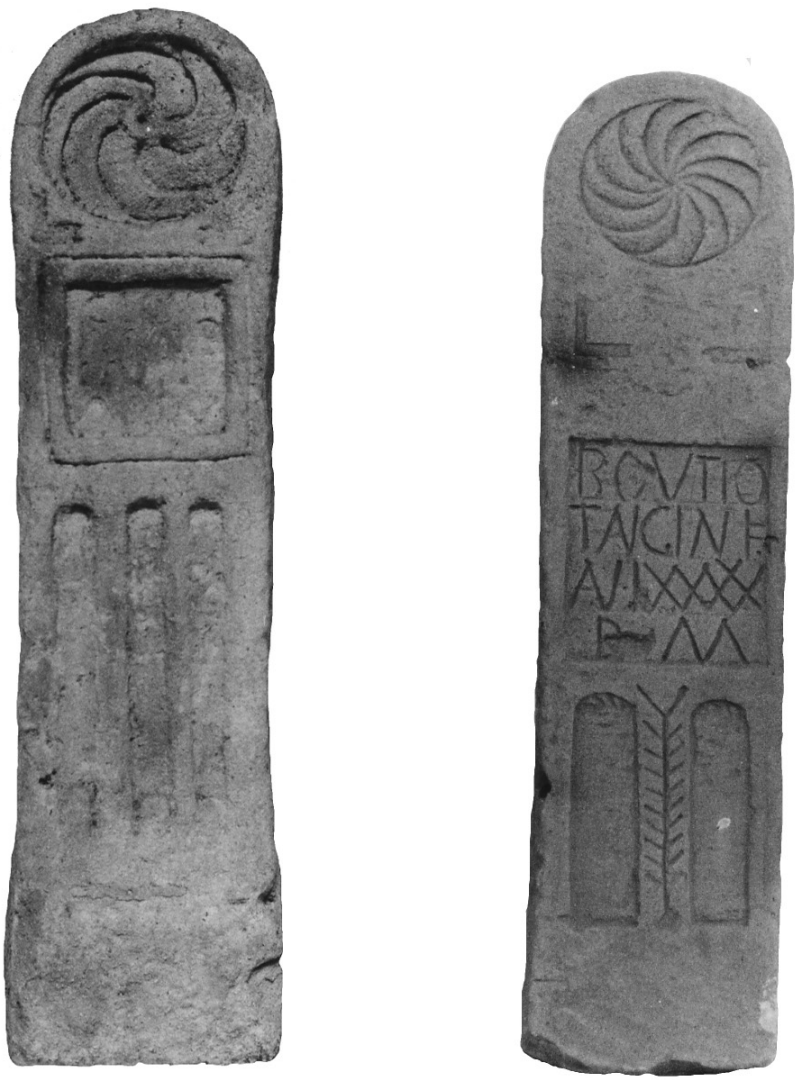

FIG. 6 - Stèles funèraires à "roue solaire" et arches (Musée provincial de Salamanque 

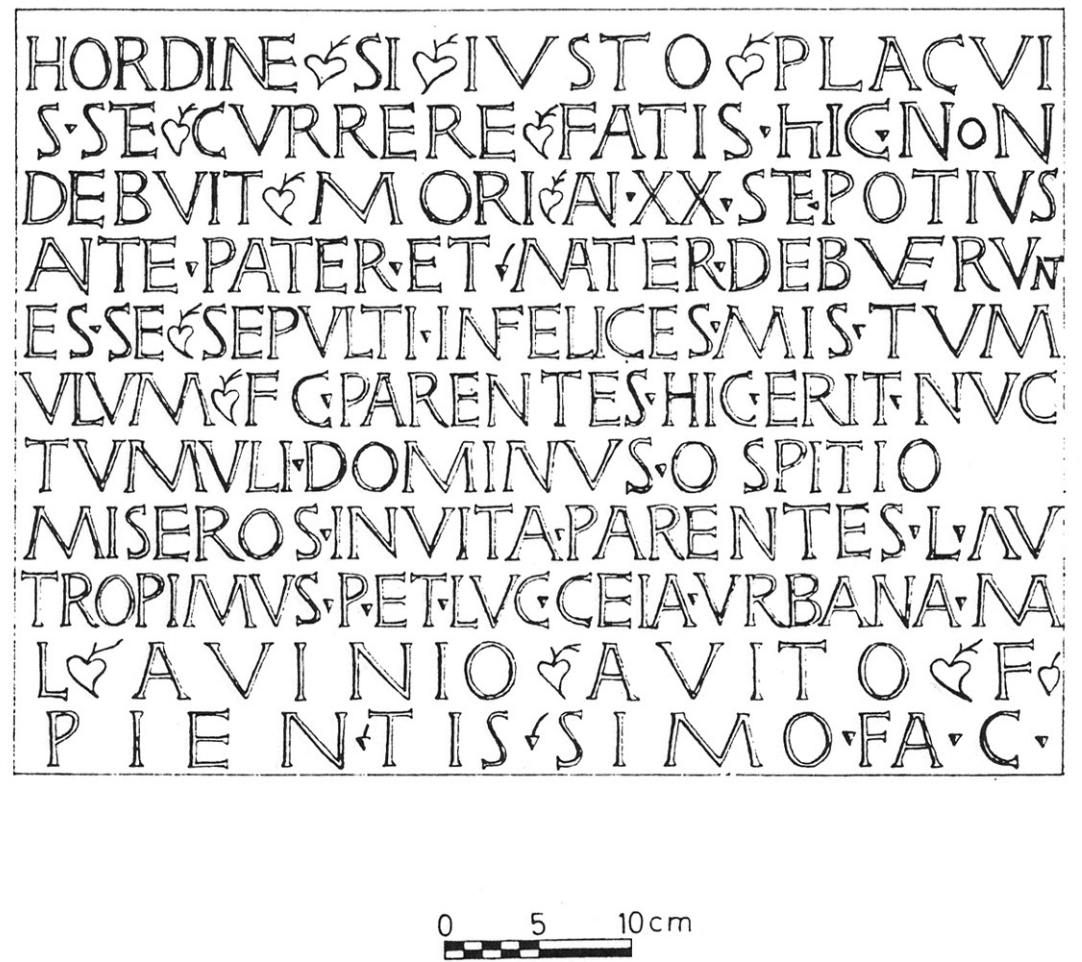

FIG. 7-Inscription funeraire de L. Avinius Avitus (Alcolea de Tajo) d'après R. Martin Valls "Inscriptión romana del Bercial, Alcolea de Tajo (Toledo), BSSA, XXXVII, 1971, 425-429. 


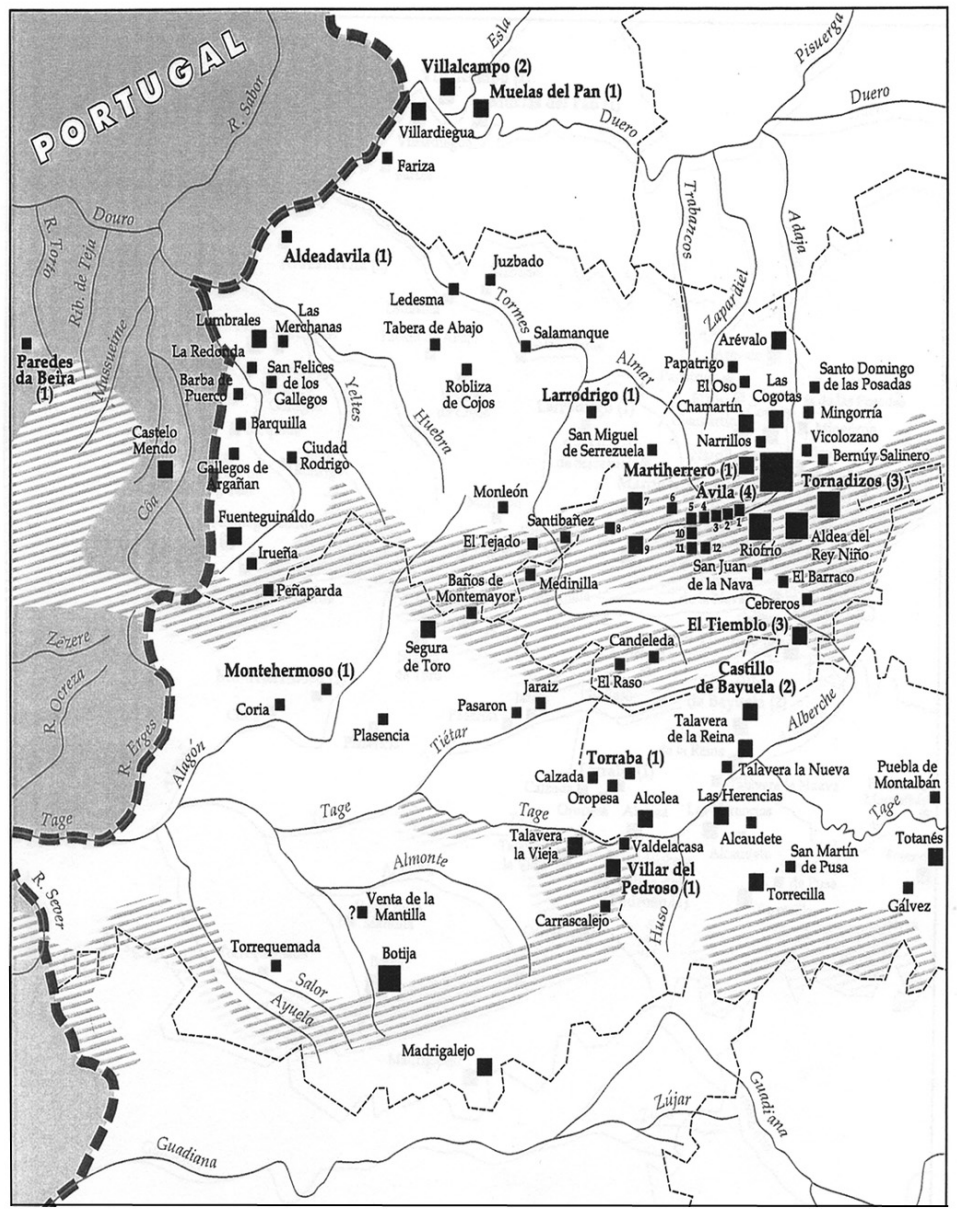

1: La Colilla

2: Padiernos

0

3 : Muñochas

$25 \mathrm{~km}$

Limite de province actuelle

Zone montagneuse

4 : Muñogalindo

5 : Santa María del Arroyo

6: Muraña

7: Villanueva del Campillo

8: Bonilla

9: Villatoro

10: Solosancho

11: Villaviciosa

12: Sotalvo

\section{Nombre de sculptures}

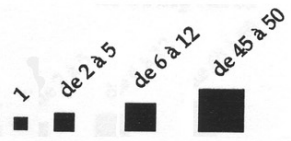

Aldeadavila : verracos gravés d'une inscription latine

(5): Nombre de verracos gravés d'une inscription latine

Fig. 8- Les sculptures zoomorphes 

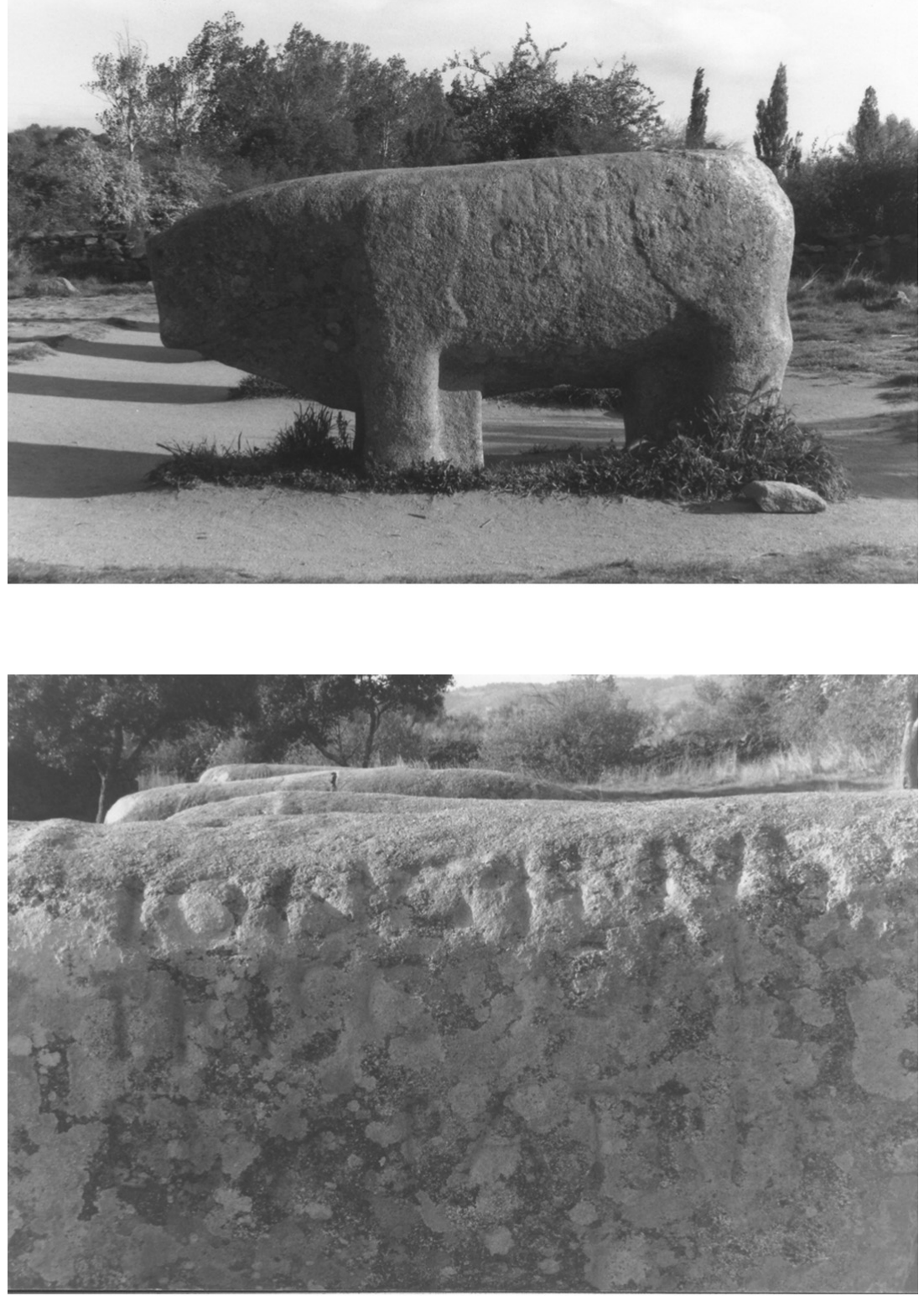

FIG. 9 - Sculptures zoomorphes de Guisando (province d'Àvila) 

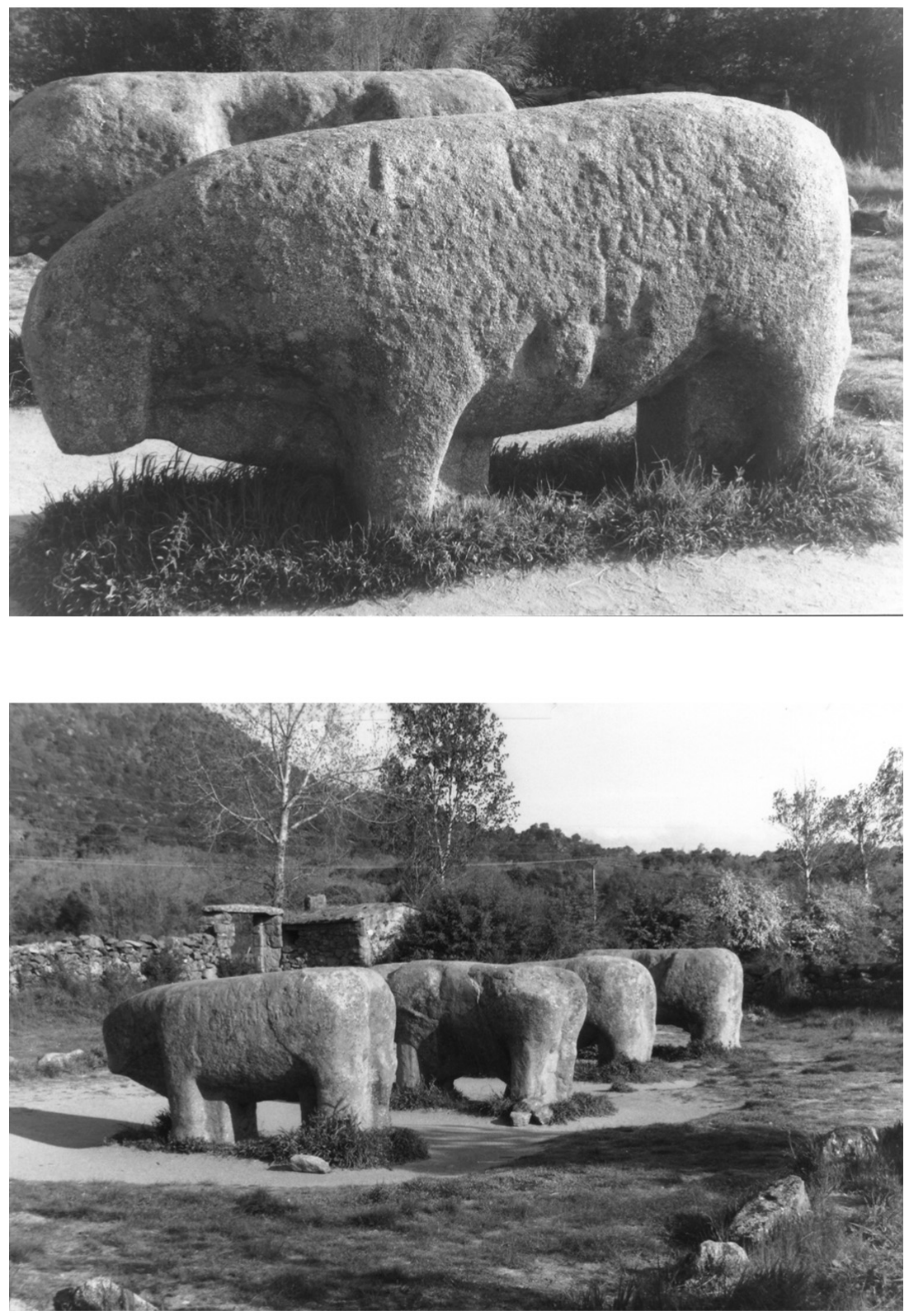

FIG. 9A- Sculptures zoomorphes de Guisando (province d'Àvila) 